\title{
Feeding Ecology and Host Preferences of Mosquitoes in two Zoological Gardens in the United Kingdom
}

\author{
Arturo Hemandez-Colina ( $\nabla$ taurendil9@hotmail.com ) \\ The University of Liverpool https://orcid.org/0000-0002-4482-5886 \\ Merit Gonzalez-Olvera \\ University of Liverpool Institute of Infection and Global Health \\ Emily Lomax \\ University of Liverpool Institute of Infection and Global Health \\ Freya Townsend \\ University of Liverpool Institute of Infection and Global Health \\ Amber Maddox \\ University of Liverpool Institute of Infection and Global Health \\ Jenny C. Hesson \\ Uppsala University Department of Medical Biochemestry and Microbiology \\ Kenneth Sherlock \\ University of Liverpool Institute of Infection and Global Health \\ Dawn Ward \\ Flamingo Land \\ Lindsay Eckley \\ North of England Zoological Society \\ Javier Lopez \\ North of England Zoological Society \\ Matthew Baylis \\ University of Liverpool Institute of Infection and Global Health
}

\section{Research}

Keywords: bloodmeal, Culex pipiens, Culiseta annulata, mosquito control, mosquito dispersal

Posted Date: December 4th, 2020

DOl: https://doi.org/10.21203/rs.3.rs-117765/v1

License: (c) (1) This work is licensed under a Creative Commons Attribution 4.0 International License. Read Full License 


\section{Abstract}

Background: Zoological gardens contain unique configurations of exotic and endemic animals and plants that create a diverse range of developing sites and potential sources of blood meals for local mosquitoes. This may imply unusual interspecific pathogen transmission risks involving zoo animals, like avian malaria to captive penguins. Understanding mosquito ecology and host preferences is necessary to improve mosquito control and disease prevention measures in these environments.

Methods: Mosquito sampling took place in Chester Zoo for three years (2017 to 2019) and for one year in Flamingo Land (2017) using different trapping methods. Blood-fed mosquitoes were identified and their bloodmeal was amplified by PCR, sequenced, and blasted for host species identification.

Results: In total, 640 blood-fed mosquitoes were collected (Culex pipiens $(\mathrm{n}=497)$, Culiseta annulata $(\mathrm{n}=81)$, Anopheles maculipennis s.l. ( $\mathrm{n}=7)$, An. claviger $(n=1)$ and unidentifiable $(n=55)$ ). Successful identification of the host species was achieved from 159 of the 640 blood-fed mosquitoes. Mosquitoes fed on birds $(n=74)$, non-human mammals $(n=20)$ and humans $(n=71)$. There were mixed bloodmeals from two hosts $(n=6)$. The proportions of blood-fed mosquitoes varied across sampling seasons and sites within the zoos. The use of resting traps and aspiration of vegetation were more efficient techniques for capturing blood-fed mosquitoes than traps for host-seeking or gravid mosquitoes. By relating the locations of zoo animals to where fed mosquitoes were trapped, the minimum travelling distances were calculated (13.7 to 366.7 meters). Temperature, precipitation, relative humidity, proximity to zoo animal exhibits and vegetation level were found to be significantly associated with the proportion of captured blood-fed mosquitoes by generalized linear modelling.

Conclusions: Mosquito feeding behaviour in zoos is influenced by environmental variables and host availability, which highlights the value of mosquito monitoring in complex settings to plan control strategies and potentially reduce inherent disease transmission risks for humans and threatened zoo animals.

\section{Background}

In zoological gardens, mosquitoes have access to a broad range of hosts and breeding sites due to the combination of endemic and exotic species of fauna and flora that create the zoo environment [1]. Mosquitoes collected at zoos have been found feeding on zoo animals, free wild animals, and humans, and mixed blood-meals have been reported [2-4]. The zoo environment poses a risk of interspecific transmission of pathogens among vertebrate groups that usually cannot happen in natural environments [1]. The relevance of mosquito ecology in zoos can be exemplified by the outbreak of West Nile virus (WNV), transmitted by Culex spp. mosquitoes, in the Bronx Zoo/Wildlife Conservation Park in relation to the first outbreak of WNV in America in humans [5]. The first fully sequenced strain of WNV in North America was isolated from a flamingo kept at this zoo [6]. Furthermore, it has been observed that host preference changes in Culex pipiens between humans and birds at different times in the season could be related to the infection risk with WNV for humans [7]. Mosquito-borne diseases can be also a threat for the health of zoo animals; avian malaria (caused by Plasmodium spp. haemosporidians) clearly exemplifies this as it is a major cause of severe disease and death for captive penguins worldwide [8, 9]. Moreover, as the parasite and its mosquito vectors have a broad distribution, this infection should be considered as a constant threat and regular surveillance of mosquitoes in zoos has been encouraged [10].

The choice or availability of hosts on which mosquitoes feed is an important factor of the ability of mosquito populations to transmit pathogens, a measure known as 'vectorial capacity'. Different mosquito species can exhibit diverse feeding patterns despite being exposed to the same vertebrates in a single location [11]. Mosquitoes can be generalists, feeding on a wide range of species or groups of vertebrates, or specialists, preferring a narrow range of host species or types $[12,13]$. Generalist mosquitoes can facilitate pathogen transmission among unrelated species (for example, birds and mammals) and in such cases, they are known as 'bridge vectors' [14-17]. However, the feeding of mosquitoes on hosts than cannot harbour the pathogen may lead to a dilution effect in pathogen transmission [2]. In the case of specialized mosquitoes, changes in the host community structure could force them to feed on unusual hosts [14] which could also facilitate interspecific transmission of pathogens. For example, Aedes japonicus that usually feeds on mammals (mammalophilic) has been found to feed on birds in nature [18]. Likewise, when the mammalian-biting biotype of Culex pipiens (molestus) and its birdfeeding biotype (pipiens) hybridize, they resulting population may present mixed host preferences and therefore, could act as a bridge vector for pathogens from birds to people [15]. Therefore, both, generalist and specialist mosquitoes can function as bridge vectors under certain circumstances, and the combination of vector's host preference and host competence determine if cross-species transmission occurs and whether a dilution or amplification effect is observed in relation to host diversity [19].

Mosquito dispersal is another factor related to disease transmission risks. Female mosquitoes can travel long distances when seeking a blood-meal. Some species, like Aedes vexans, migrate to invade new habitats [20] and Culex spp. for example, can disperse for several kilometres [21]. Previous studies on flying distances of blood-fed mosquitoes in zoos have proved that they feed on captive animals, mostly travel short distances and are implicated in the local transmission of vector-borne diseases to zoo animals [4, 22].

Here, the feeding ecology, host preferences and dispersal of mosquitoes in two zoological gardens in the UK are described to better understand the dynamics of mosquito populations and get insights into the potential for cross-species transmission of pathogens. Over two years, we identified bloodmeals from mosquitoes captured using traps for host-seeking mosquitoes and for gravid mosquitoes looking to oviposit; special techniques for capturing blood-engorged mosquitoes were implemented in a third sampling year in one zoological garden. As mosquitoes are highly adaptable, this information is relevant for planning mosquito control strategies in modified environments and to protect vulnerable hosts. 


\section{Methods}

\section{Sites and sampling methods}

We performed general sampling of mosquitoes for two years (2017 and 2018) in Chester Zoo (Upton by Chester, Cheshire, UK) and for one year (2017) in Flamingo Land (Kirby Misperton, North Yorkshire, UK). In 2019, we specifically sampled for blood-fed mosquitoes in Chester Zoo only.

The traps that we used in all samplings were the BG-Mosquitaire trap and the CDC-Gravid trap model 1712. The BG-Mosquitaire trap has a fan inside a plastic container that is connected to AC power; this trap captures host-seeking mosquitoes with its basic attractant, the BG-Sweetscent ${ }^{\circledR}$, based on lactic acid to mimic mammalian sweat [23]. The CDC-Gravid trap model 1712 consists of an electric fan powered by a $6 \mathrm{~V}$ battery located inside a plastic cylinder and covered with a capture net, which is placed over a tray that contains $4 \mathrm{~L}$ of infusion media as attractant for egg laying mosquitoes [24]. The infusion media is prepared with tap water $(40 \mathrm{~L})$, hay $(200 \mathrm{gr})$, brewer's yeast $(2 \mathrm{gr})$ and milk powder $(2 \mathrm{gr})$, and the mix is rested for at least one week before use [25].

For the 2019 season, four capture methods were used: BG-Mosquitaire trap upgraded with dry ice as source of $\mathrm{CO}_{2}, \mathrm{CDC}^{-G r a v i d}$ trap, resting trap, and aspiration in mosquito resting areas. The BG-Mosquitaire traps operated using the basic attractant (BG-Sweetscent $\left.{ }^{\circledR}\right)$ and dry ice to increase the chances of capturing host-seeking mosquitoes and the species range [26]. For this, a polyurethane box was placed next to each trap, connected to the trap with a plastic tube and sealed with silicone glue; the lid was sealed to the box with tape and a strap with a combination lock to prevent accidental opening. The CDC-Gravid traps were used as described previously. The resting trap is a $40 \times 30 \times 30 \mathrm{~cm}$ wooden box with an open side which provides shelter; there is no attractant used, mosquitoes simply go inside the box to rest, especially during daytime. We placed these traps with the open face on the underside, leaning against a structure (e.g. walls, trees, or rocks) at $45^{\circ}$ to allow a gap for mosquito entry. We used an Improved CDC-Backpack Aspirator model 1412 [27] to aspirate the inside of the resting traps. Finally, the same aspirator was used to systematically aspirate potential resting places, such as vegetation, walls, fences, and the outside of buildings, for five minutes in each sampling area (Fig. 1).

Sampling areas were defined as $30 \mathrm{~m}$ diameter circles that contained one trap of each type, and set in public areas, staff areas or animal exhibits. Inside sampling areas, the traps were at least $10 \mathrm{~m}$ apart from each other to minimize interference between them, and located considering safety for people and animals, logistic implications, surrounding vegetation (avoiding coverage at least $2 \mathrm{~m}$ above them to reduce the possibility of leaves interfering with the trap's fan), and with protection from direct sunlight and rain when possible.

\section{Sampling protocols}

We established ten sampling areas in Chester Zoo in the 2017 season; in 2018 we discontinued three areas due to low catches, and added a new area located inside the penguin exhibit (Fig. 2). Sampling took place for 32 weeks in Chester Zoo in 2017 (May to December) and for 31 in 2018 (April to November). In Flamingo Land we established four sampling areas (Fig. 3), which were sampled for 25 weeks in 2017 (June to November). In both sites, BG-Mosquitaire traps were emptied once a week after being operational for six consecutive nights and once a week after operating for one night; the CDCGravid traps were operated one night per week. This resulted in a six day collections from the BG-Mosquitaire traps, followed by a one day collection from both traps every week.

In addition, specific sampling of blood-fed mosquitoes took place in Chester Zoo for 11 weeks (April to July) in 2019 using five sampling areas close to the penguin exhibit (Fig. 2). In this occasion, the weekly schedule consisted of one collection from the BG-Mosquitaire traps after five days with the basic attractant plus two collections after one day each adding dry ice, two aspirations from resting traps and two from surrounding areas, and one collection from the CDC-Gravid traps after two days of operation.

\section{Environment monitoring}

The environment was monitored for landscape and weather variables. Landscape variables were categorized with three possible values and included vegetation (dense, medium, or scarce), proximity to suitable mosquito oviposition sites (close, medium, or remote), mosquito resting areas (abundant, medium or rare) and proximity to zoo animal exhibits (close, medium, or remote). The vegetation value was changed to scarce for all traps when foliage decreased in autumn. The definition of variable values can be found in supplemental material Table S1 and the corresponding values per trap in Table S2.

Weather variables included regional temperature, relative humidity, wind speed and precipitation. Daily values were obtained using the R package "Climate", which extracts information from the OGIMET Weather Information Service (www.ogimet.com). We downloaded data from the closest inland weather stations; for Chester Zoo it is Hawarden ( $13 \mathrm{~km}$ ) and for Flamingo Land, Linton On Ouse (52.2 km). We did not use the data from closer stations to Flamingo Land because they are on the coast and, therefore, influenced by marine climate.

In 2017 and 2018 we used TinyTag@ loggers, 13 of the Plus 2 TGP-4500 model and two of the Ultra 2 TGU-4500 model, (Gemini Data Loggers, UK) to record local temperature and humidity. Loggers were placed next to the BG-Mosquitaire traps, programmed to record every hour and average readings were used. The loggers were not used in 2019.

\section{Laboratory protocols}

Collected nets were transported in a cooled icebox and placed into a $-20^{\circ} \mathrm{C}$ freezer on arrival for approximately two hours. Mosquitoes were separated from accompanying insects and identified by morphology under a stereomicroscope following identification keys [20, 28]. During the morphological identification, the abdomens of the blood-fed mosquitoes were cut off using entomological forceps and disposable scalpels; the materials were cleaned 
with $70 \%$ ethanol and DNA Away® between specimens. The abdomens were stored in individual reaction tubes at $-80{ }^{\circ} \mathrm{C}$ for no more than three weeks until processing. As field captured mosquitoes have different degrees of blood digestion that affects host identification [29], mosquitoes were selected for analysis if they were engorged with a red, dark red or blackish abdomen indicating blood content. This is equivalent to the stages II and III of the Sella classification system [30, 31].

Mosquito abdomens were homogenized with $200 \mu \mathrm{l}$ of PBS (phosphate buffered saline) per sample [32] using sterile plastic pestles or with a stainlesssteel bead and a QIAGEN@ Tissuelysser at frequency of $24 \mathrm{~Hz}$ per second for 2 minutes. For DNA extraction, we used the OMEGA Bio-Tek E.Z.N.A ${ }^{\circledR}$ Tissue DNA kit following the manufacturer's instructions; extracts were stored at $4{ }^{\circ} \mathrm{C}$ until further processing for no more than two weeks and at $-20{ }^{\circ} \mathrm{C}$ for long term storage. Afterwards, the PCR protocol and primers proposed by Alcaide et al. [33] were used to obtain a 758 bp amplicon of the Cytochrome $c$ Oxidase Subunit I (COI) gene. Negative controls (nuclease-free water) were added every five samples and DNA extract from liver of Black headed gull (Larus ridibundus) was used as a positive control. The amplification was verified by electrophoresis in a $1 \%$ agarose gel. Positive PCR products were sent to Macrogen Europe B. V. for Sanger sequencing using the M13 primer in the forward direction. All the PCR negative samples were tested at least twice.

Successful sequences were edited and analysed using the software BioEditC and compared to the reported sequences in the Basic Local Alignment Search Tool (BLAST®), optimized for the highly similar sequences, and the Barcoding of Life Data System $\odot$ (BOLD). The most similar sequences were aligned and compared using BioEdit@ to find the best match considering the identity and query covers and excluding wild native species absent in the area and exotic species not included in the zoo collections. With the same software, we inspected the electropherograms for double peaks in a single base position, which, if consistent, indicate that the blood-meal was mixed, with the mosquito having fed on more than one host species [33].

Mosquitoes belonging to the Culex spp. genus include, in the UK, two sympatric species that are indistinguishable morphologically, $C x$. pipiens and $C x$. torrentium. Females from this genus were identified using the PCR and enzyme restriction protocol developed by Hesson et al. [34]. Likewise, $C x$. pipiens has two morphologically identical biotypes with different biological traits and epidemiologic roles, pipiens and molestus; the first one of which is reported predominately as ornithophilic and the second as mammalophilic [15, 35, 36]. We found $C x$. pipiens feeding on humans, so we tested them using the multiplex PCR protocol proposed by Bahnck et al. [37] to differentiate their biotype.

\section{Flying Distances}

Knowing the zoo animals on which mosquitoes have fed, we estimated the distance between the relevant animal exhibits and the location of the trap where the blood-fed mosquitoes were captured. The software Species360 ZIMS (Zoological Information Management Software) [38] was used to determine the location of the zoo animals at the time when mosquitoes were captured. The Open Street Map layer was used in the QGIS 3.2C software to delineate the polygons of the exhibits. Then, the centroid of the exhibits was estimated, and the minimum travelling distance of mosquitoes was represented as the length of the line from the centroid to the corresponding trap.

\section{Data analysis}

A gross analysis comprised the comparison of blood-fed mosquito proportions separately, by zoo and sampling year, to detect differences by sampling area, month, and capture method. Host preferences were compared by vertebrate group (birds, non-human mammals, and humans) and mosquito species using the Chi-squared test of independence exploring the test residuals to find sources of significance. Fisher's exact test of independence was used when appropriate. Specimens with damaged or missing abdomens were excluded and only completely identified mosquitoes were included in the analyses by species.

To understand environmental influences in blood-fed mosquito captures, we used generalized linear models (GLMs) for a proportion response using a binomial family (or quasibinomial in case of overdispersion) and a logit link, with a backwards elimination process to find minimal adequate models. Mosquito data was consolidated per week and weather data was averaged for the week before collection. The models were constructed for each sampling separately including weather and landscape variables, and for the overall mosquito collection using weather variables. Regional and local temperature data was expected to be strongly correlated so the one producing the best model fit was used. Models were constructed using the MASS package of the $\mathrm{R}$ software [39].

\section{Results}

Excluding males and mosquitoes with damaged abdomens, the number of blood-fed mosquitoes captured (and percentage of all female mosquitoes caught) in Chester Zoo was 213 (3.5\%) in 2017, 245 (9.7\%) in 2018 and 107 (4.1\%) in 2019. We caught 75 (7.2\%) in Flamingo Land in 2017. In total, we caught 640 blood-fed mosquitoes (5.2\%) (Table 1). Most blood-fed mosquitoes in both sites were Culex pipiens $(\mathrm{n}=497)$ and Culiseta annulata $(\mathrm{n}=81)$, although we also captured one Anopheles claviger and four An. maculipennis s.l. in 2018 and three An. maculipennis s.l. in 2019. Fifty-five mosquitoes were damaged and unable to be identified to species level. PCR testing confirmed that all engorged Culex spp. mosquitoes were $C x$. pipiens and all of those which fed on humans were $C x$. pipiens biotype pipiens. Other species captured include Aedes annulipes, Ae. vexans, Ae. detritus, An. plumbeus, An. claviger, Cs. morsitans and $C x$. torrentium, although these mosquitoes were not blood-fed and captured in low numbers. 
Table 1

Blood-fed mosquitoes captured and testing results per sampling.

\begin{tabular}{|c|c|c|c|c|c|c|c|c|c|c|c|c|}
\hline \multirow[t]{2}{*}{ Sampling } & \multicolumn{5}{|c|}{ Blood-feds / Total captureda (\%) } & \multirow{2}{*}{$\begin{array}{l}\text { PCR } \\
\text { positives } \\
(\%)^{b}\end{array}$} & \multirow{2}{*}{$\begin{array}{l}\text { Sequencing } \\
\text { matches } \\
(\%)^{c}\end{array}$} & \multicolumn{5}{|c|}{ Identified Hosts (\%) ${ }^{d}$} \\
\hline & $\begin{array}{l}\text { BG- } \\
\text { Mosquitaire }\end{array}$ & $\begin{array}{l}\text { CDC- } \\
\text { Gravid }\end{array}$ & $\begin{array}{l}\text { Resting } \\
\text { Trap }\end{array}$ & Aspiration & Total & & & Birds & $\begin{array}{l}\text { Non- } \\
\text { human } \\
\text { mammals }\end{array}$ & Humans & Total & $\begin{array}{l}\text { Mixed } \\
\text { hosts }\end{array}$ \\
\hline $\begin{array}{l}\text { Chester } \\
\text { Zoo } 2017\end{array}$ & $\begin{array}{l}132 / 3424 \\
(3.9)\end{array}$ & $\begin{array}{l}81 / \\
2593 \\
(3.1)\end{array}$ & - & - & $\begin{array}{l}213 / \\
6017 \\
(3.5)\end{array}$ & $\begin{array}{l}95 \\
(44.6)\end{array}$ & $\begin{array}{l}75 \\
(78.9)\end{array}$ & $\begin{array}{l}31 \\
(38.8)\end{array}$ & $\begin{array}{l}9 \\
(11.3)\end{array}$ & $\begin{array}{l}40 \\
(50.0)\end{array}$ & $\begin{array}{l}80 \\
(100)\end{array}$ & $\begin{array}{l}5 \\
(6.6)\end{array}$ \\
\hline $\begin{array}{l}\text { Chester } \\
\text { Zoo } 2018\end{array}$ & $\begin{array}{l}185 / 1988 \\
(9.3)\end{array}$ & $\begin{array}{l}60 / \\
545 \\
(11)\end{array}$ & - & - & $\begin{array}{l}245 / \\
2533 \\
(9.7)\end{array}$ & $\begin{array}{l}56 \\
(22.9)\end{array}$ & $\begin{array}{l}45 \\
(80.4)\end{array}$ & $\begin{array}{l}24 \\
(52.2)\end{array}$ & $\begin{array}{l}5 \\
(10.9)\end{array}$ & $\begin{array}{l}17 \\
(37.0)\end{array}$ & $\begin{array}{l}46 \\
(100)\end{array}$ & $\begin{array}{l}1 \\
(2.2)\end{array}$ \\
\hline $\begin{array}{l}\text { Chester } \\
\text { Zoo } 2019\end{array}$ & $\begin{array}{l}39 / 1505 \\
(2.6)^{\mathrm{e}}\end{array}$ & $\begin{array}{l}43 / \\
971 \\
(4.4)\end{array}$ & $\begin{array}{l}12 / 65 \\
(18.5)\end{array}$ & $\begin{array}{l}13 / 59 \\
(22)\end{array}$ & $\begin{array}{l}107 / \\
2600 \\
(4.1)\end{array}$ & $\begin{array}{l}23 \\
(21.5)\end{array}$ & $\begin{array}{l}20 \\
(86.9)\end{array}$ & $\begin{array}{l}18 \\
(90.0)\end{array}$ & $\begin{array}{l}0 \\
(0)\end{array}$ & $\begin{array}{l}2 \\
(10.0)\end{array}$ & $\begin{array}{l}20 \\
(100)\end{array}$ & $\begin{array}{l}0 \\
(0)\end{array}$ \\
\hline $\begin{array}{l}\text { Flamingo } \\
\text { Land } \\
2017\end{array}$ & $\begin{array}{l}46 / 862 \\
(5.3)\end{array}$ & $\begin{array}{l}29 / \\
186 \\
(15.6)\end{array}$ & - & - & $\begin{array}{l}75 / \\
1048 \\
(7.2)\end{array}$ & $\begin{array}{l}30 \\
(40)\end{array}$ & $\begin{array}{l}19 \\
(63.3)\end{array}$ & $\begin{array}{l}1 \\
(5.3)\end{array}$ & $\begin{array}{l}6 \\
(31.6)\end{array}$ & $\begin{array}{l}12 \\
(63.2)\end{array}$ & $\begin{array}{l}19 \\
(100)\end{array}$ & $\begin{array}{l}0 \\
(0)\end{array}$ \\
\hline Total & $\begin{array}{l}402 / 7779 \\
(5.2)\end{array}$ & $\begin{array}{l}213 / \\
4295 \\
(4.9)\end{array}$ & $\begin{array}{l}12 / 65 \\
(18.5)\end{array}$ & $\begin{array}{l}13 / 59 \\
(22)\end{array}$ & $\begin{array}{l}640 / \\
12198 \\
(5.2)\end{array}$ & $\begin{array}{l}204 \\
(31.9)\end{array}$ & $\begin{array}{l}159 \\
(77.9)\end{array}$ & $\begin{array}{l}74 \\
(44.8)\end{array}$ & $\begin{array}{l}20 \\
(12.1)\end{array}$ & $\begin{array}{l}71 \\
(43.0)\end{array}$ & $\begin{array}{l}165 \\
(100)\end{array}$ & $\begin{array}{l}6 \\
(3.6)\end{array}$ \\
\hline
\end{tabular}

\section{Methods assessment}

Proportions of blood-fed mosquitoes were compared by traps, excluding females with abdominal damage, and using only the one-day collections in the case of the BG-Mosquitaire traps. There were no significant differences either in Chester Zoo in $2017\left(X^{2}=0.047, d f=1, P=0.829\right)$ or 2018 $\left(X^{2}=1.373\right.$, df $=1, P=0.241)$ or Flamingo Land $\left(X^{2}=2.688, d f=1, P=0.101\right)$. Nonetheless, we observed a significant difference comparing all methods used in 2019 (Fisher's exact test, $\mathrm{P}<0.0001$ ): a higher proportion of blood-fed mosquitoes were captured in resting traps and by aspiration than by BG-Mosquitaire traps or CDC-gravid traps. The success of PCR amplification per sampling varied from 21.5 to $44.6 \%$ and was $31.9 \%$ overall. From these samples, the sequencing success varied from 63.3 to $86.9 \%$ being $77.9 \%$ overall (Table 1 ).

\section{Host preferences}

The vertebrate hosts were identified from 159 blood-fed mosquitoes, and were grouped as birds $(n=74)$, non-human mammals $(n=20)$, and humans $(n=$ 71); two different hosts were identified from the same mosquito in six occasions which were counted as a preference for both hosts (Table 1). The most common species of free wild birds were passerine residents, Eurasian jackdaw (Corvus monedula) $(\mathrm{n}=11)$, Eurasian magpie $($ Pica pica) $(\mathrm{n}=9)$ and Eurasian blackbird (Turdus merula) $(\mathrm{n}=8)$; although Mallard (Anas platyrhynchos), another resident (order Anseriformes), was also common ( $\mathrm{n}=7)$. From the five species of zoo birds identified, the Schalow's turaco (Tauraco schalowi) $(\mathrm{n}=5)$, Humboldt penguin (Spheniscus humboldti) ( $\mathrm{n}=4)$ and Javan green magpie (Cissa thalassina) $(\mathrm{n}=2)$ were more frequently identified. The zoo non-human mammals most often found were the Bactrian camel (Camelus bactrianus) $(n=6)$, Eld's deer (Rucervus eldii thamin) $(n=3)$, Scimitar-horned oryx (Oryx dammah) $(n=2)$ and capybara $($ Hydrochoerus hydrochaeris) $(\mathrm{n}=2)$.

In Chester Zoo, eight species of free wild birds, two zoo birds, four zoo mammals, one cattle and 37 samples from human were identified in 2017 (Table 2). The following year, we found nine species of free wild birds, two zoo birds, one chicken, one cattle, one pig, two zoo mammals and 17 humans (Table 3). In 2019, we identified five species of free wild birds, two zoo birds and two humans (Table 4). In Flamingo Land (2017), one free wild bird, three species of zoo mammals, one dog and 10 humans were identified (Table 5). Proportions of hosts are shown overall per sampling in Fig. 4 , for $C x$. pipiens in Fig. 5 and for the other species in supplementary material Fig. S1. 
Table 2

Host preferences of blood-fed mosquitoes captured in Chester Zoo, 2017

\begin{tabular}{|c|c|c|c|c|c|c|}
\hline Host Group & Scientific Name & Common name & $\begin{array}{l}\text { Cx. } \\
\text { pipiens } \\
(\%)\end{array}$ & $\begin{array}{l}\text { Cs. } \\
\text { annulata } \\
(\%)\end{array}$ & $\begin{array}{l}\text { Unknown* } \\
\text { (\%) }\end{array}$ & $\begin{array}{l}\text { Total } \\
\text { (\%) }\end{array}$ \\
\hline \multirow{10}{*}{$\begin{array}{l}\text { Birds } \\
(n=26)\end{array}$} & Anas platyrhynchos & Mallard & $1(1.3)$ & $0(0.0)$ & $0(0.0)$ & $1(1.3)$ \\
\hline & Cissa thalassina & Javan green magpie ${ }^{z}$ & $2(2.7)$ & $0(0.0)$ & $0(0.0)$ & $2(2.7)$ \\
\hline & Corvus monedula & Eurasian jackdaw & $1(1.3)$ & $0(0.0)$ & $0(0.0)$ & $1(1.3)$ \\
\hline & Passer domesticus & House sparrow & $3(4.0)$ & $0(0.0)$ & $0(0.0)$ & $3(4.0)$ \\
\hline & Pica pica & Eurasian magpie & $4(5.3)$ & $1(1.3)$ & $1(1.3)$ & $6(8.0)$ \\
\hline & Prunella modularis & Dunnock & $1(1.3)$ & $0(0.0)$ & $0(0.0)$ & $1(1.3)$ \\
\hline & Pyrrhula pyrrhula & Eurasian bullfinch & $1(1.3)$ & $0(0.0)$ & $0(0.0)$ & $1(1.3)$ \\
\hline & Tauraco schalowi & Schalow's turaco z & $3(4.0)$ & $0(0.0)$ & $1(1.3)$ & $4(5.3)$ \\
\hline & Turdus merula & Eurasian blackbird & $5(6.7)$ & $0(0.0)$ & $1(1.3)$ & $6(8.0)$ \\
\hline & Turdus philomelos & Song thrush & $1(1.3)$ & $0(0.0)$ & $0(0.0)$ & $1(1.3)$ \\
\hline \multirow{4}{*}{$\begin{array}{l}\text { Mammals } \\
(\mathrm{n}=7)\end{array}$} & Bos taurus & Cattle & $0(0.0)$ & $1(1.3)$ & $0(0.0)$ & $1(1.3)$ \\
\hline & Camelus bactrianus & Bactrian camel ${ }^{z}$ & $0(0.0)$ & $3(4.0)$ & $0(0.0)$ & $3(4.0)$ \\
\hline & Rucervus eldii thamin & Eld's deer $\mathrm{z}$ & $0(0.0)$ & $2(2.7)$ & $0(0.0)$ & $2(2.7)$ \\
\hline & Tragelaphus eurycerus & Bongo $^{z}$ & $0(0.0)$ & $1(1.3)$ & $0(0.0)$ & $1(1.3)$ \\
\hline $\begin{array}{l}\text { Humans } \\
(\mathrm{n}=37)\end{array}$ & Homo sapiens & Human & $27(36.0)$ & $9(12.0)$ & $1(1.3)$ & $\begin{array}{l}37 \\
(49.3)\end{array}$ \\
\hline $\begin{array}{l}\text { Mixed blood- } \\
\text { meals }\end{array}$ & $\begin{array}{l}\text { Camelus bactrianus / Turdus } \\
\text { merula }\end{array}$ & Bactrian camel $\mathrm{z} /$ Eurasian blackbird & $1(1.3)$ & $0(0.0)$ & $0(0.0)$ & $1(1.3)$ \\
\hline \multirow[t]{4}{*}{$(n=5)$} & $\begin{array}{l}\text { Giraffa camelopardalis } \\
\text { rothschildi / }\end{array}$ & $\begin{array}{l}\text { Rothschild's giraffe }{ }^{z} / \text { Schalow's } \\
\text { turaco }^{z}\end{array}$ & $1(1.3)$ & $0(0.0)$ & $0(0.0)$ & $1(1.3)$ \\
\hline & Tauraco schalowi & & & & & \\
\hline & $\begin{array}{l}\text { Pica pica / } \\
\text { Homo sapiens }\end{array}$ & Eurasian magpie / Human & $3(4.0)$ & $0(0.0)$ & $0(0.0)$ & $3(4.0)$ \\
\hline & & Total & $54(72.0)$ & $17(22.7)$ & $4(5.3)$ & $\begin{array}{l}75 \\
(100.0)\end{array}$ \\
\hline
\end{tabular}


Table 3

Host preferences of blood-fed mosquitoes captured in Chester Zoo, 2018.

\begin{tabular}{|c|c|c|c|c|c|c|c|}
\hline Host group & Scientific Name & Common name & $\begin{array}{l}\text { Cx. } \\
\text { pipiens } \\
(\%)\end{array}$ & $\begin{array}{l}\text { Culiseta } \\
\text { spp. (\%) }\end{array}$ & $\begin{array}{l}\text { Anopheles } \\
\text { maculipennis s.l. (\%) }\end{array}$ & $\begin{array}{l}\text { Unknown* } \\
(\%)\end{array}$ & $\begin{array}{l}\text { Total } \\
(\%)\end{array}$ \\
\hline \multirow{10}{*}{$\begin{array}{l}\text { Birds } \\
(n=22)\end{array}$} & Anas platyrhynchos & Mallard & $4(8.9)$ & $2(4.4)$ & $0(0.0)$ & $0(0.0)$ & $\begin{array}{l}6 \\
(13.3)\end{array}$ \\
\hline & Gallus gallus & Chicken & $2(4.4)$ & $1(2.2)$ & $0(0.0)$ & $1(2.2)$ & $\begin{array}{l}4 \\
(8.9)\end{array}$ \\
\hline & Cyanistes caeruleus & Blue tit & $3(6.7)$ & $0(0.0)$ & $0(0.0)$ & $0(0.0)$ & $\begin{array}{l}3 \\
(6.7)\end{array}$ \\
\hline & Passer domesticus & House sparrow & $2(4.4)$ & $0(0.0)$ & $0(0.0)$ & $0(0.0)$ & $\begin{array}{l}2 \\
(4.4)\end{array}$ \\
\hline & Spheniscus humboldti & Humboldt penguin ${ }^{z}$ & $0(0.0)$ & $0(0.0)$ & $1(2.2)$ & $1(2.2)$ & $\begin{array}{l}2 \\
(4.4)\end{array}$ \\
\hline & Corvus monedula & Eurasian jackdaw & $1(2.2)$ & $0(0.0)$ & $0(0.0)$ & $0(0.0)$ & $\begin{array}{l}1 \\
(2.2)\end{array}$ \\
\hline & Erithacus rubecula & European Robin & $1(2.2)$ & $0(0.0)$ & $0(0.0)$ & $0(0.0)$ & $\begin{array}{l}1 \\
(2.2)\end{array}$ \\
\hline & Strix leptogrammica & Brown wood-owl ${ }^{z}$ & $1(2.2)$ & $0(0.0)$ & $0(0.0)$ & $0(0.0)$ & $\begin{array}{l}1 \\
(2.2)\end{array}$ \\
\hline & Turdus merula & Eurasian blackbird & $1(2.2)$ & $0(0.0)$ & $0(0.0)$ & $0(0.0)$ & $\begin{array}{l}1 \\
(2.2)\end{array}$ \\
\hline & Turdus philomelos & Song thrush & $1(2.2)$ & $0(0.0)$ & $0(0.0)$ & $0(0.0)$ & $\begin{array}{l}1 \\
(2.2)\end{array}$ \\
\hline \multirow{4}{*}{$\begin{array}{l}\text { Mammals } \\
(n=5)\end{array}$} & Bos taurus & Cattle & $1(2.2)$ & $0(0.0)$ & $0(0.0)$ & $1(2.2)$ & $\begin{array}{l}2 \\
(4.4)\end{array}$ \\
\hline & Camelus bactrianus & Bactrian camel ${ }^{z}$ & $0(0.0)$ & $1(2.2)$ & $0(0.0)$ & $0(0.0)$ & $\begin{array}{l}1 \\
(2.2)\end{array}$ \\
\hline & Rucervus eldi & Eld's deer ${ }^{z}$ & $0(0.0)$ & $1(2.2)$ & $0(0.0)$ & $0(0.0)$ & $\begin{array}{l}1 \\
(2.2)\end{array}$ \\
\hline & Sus scofra & Pig & $1(2.2)$ & $0(0.0)$ & $0(0.0)$ & $0(0.0)$ & $\begin{array}{l}1 \\
(2.2)\end{array}$ \\
\hline $\begin{array}{l}\text { Humans } \\
(\mathrm{n}=17)\end{array}$ & Homo sapiens & Human & 17 (37.8) & $0(0.0)$ & $0(0.0)$ & $0(0.0)$ & $\begin{array}{l}17 \\
(37.8)\end{array}$ \\
\hline \multirow{2}{*}{$\begin{array}{l}\text { Mixed } \\
\text { blood-meals } \\
(n=1)\end{array}$} & $\begin{array}{l}\text { Columba palumbus I } \\
\text { Streptopelia decaocto }\end{array}$ & $\begin{array}{l}\text { Wood pigeon / Eurasian } \\
\text { collared dove }\end{array}$ & $1(2.2)$ & $0(0.0)$ & $0(0.0)$ & $0(0.0)$ & $\begin{array}{l}1 \\
(2.2)\end{array}$ \\
\hline & & Total & $36(80.0)$ & $5(11.1)$ & $1(2.2)$ & $3(6.7)$ & $\begin{array}{l}45 \\
(100)\end{array}$ \\
\hline
\end{tabular}


Table 4

Host preferences of blood-fed mosquitoes captured in Chester Zoo, 2019.

\begin{tabular}{|c|c|c|c|c|c|}
\hline Host group & Scientific Name & Common name & Cx. pipiens (\%) & An. maculipennis s.I. (\%) & Total (\%) \\
\hline \multirow{7}{*}{$\begin{array}{l}\text { Birds } \\
(n=18)\end{array}$} & Corvus monedula & Eurasian jackdaw & $10(50.0)$ & $0(0.0)$ & $10(50.0)$ \\
\hline & Pyrrhula pyrrhula & Eurasian bullfinch & $2(10.0)$ & $0(0.0)$ & $2(10.0)$ \\
\hline & Parus major major & Great tit & $1(5.0)$ & $0(0.0)$ & $1(5.0)$ \\
\hline & Cyanistes caeruleus & Blue tit & $1(5.0)$ & $0(0.0)$ & $1(5.0)$ \\
\hline & Gallinula chloropus & Common moorhen & $1(5.0)$ & $0(0.0)$ & $1(5.0)$ \\
\hline & Phoenicopterus ruber & Caribbean flamingo $\mathrm{z}$ & $1(5.0)$ & $0(0.0)$ & $1(5.0)$ \\
\hline & Spheniscus humboldti & Humboldt penguin z & $1(5.0)$ & $1(5.0)$ & $2(10.0)$ \\
\hline \multirow{2}{*}{$\begin{array}{l}\text { Humans } \\
(\mathrm{n}=2)\end{array}$} & Homo sapiens & Human & $1(5.0)$ & $1(5.0)$ & $2(10.0)$ \\
\hline & & Total & $18(90.0)$ & $2(5.0)$ & $20(100.0)$ \\
\hline
\end{tabular}

Table 5

Host preferences of blood-fed mosquitoes captured in Flamingo Land, 2017.

\begin{tabular}{|c|c|c|c|c|c|c|}
\hline Host Group & Scientific Name & Common name & Cx. pipiens (\%) & Culiseta spp. (\%) & Unknown* (\%) & Total (\%) \\
\hline $\begin{array}{l}\text { Birds } \\
(n=1)\end{array}$ & Parus major & Great tit & $0(0.0)$ & $0(0.0)$ & $1(5.3)$ & $1(5.3)$ \\
\hline \multirow{4}{*}{$\begin{array}{l}\text { Mammals } \\
(n=6)\end{array}$} & Camelus bactrianus & Bactrian camel $^{z}$ & $0(0.0)$ & $1(5.3)$ & $0(0.0)$ & $1(5.3)$ \\
\hline & Canis lupus familiaris & Dog & $1(5.3)$ & $0(0.0)$ & $0(0.0)$ & $1(5.3)$ \\
\hline & Hydrochoerus hydrochaeris & Capybara $^{z}$ & $1(5.3)$ & $1(5.3)$ & $0(0.0)$ & $2(10.5)$ \\
\hline & Oryx dammah & Scimitar-horned Oryx z & $0(0.0)$ & $1(5.3)$ & $1(5.3)$ & $2(10.5)$ \\
\hline \multirow{2}{*}{$\begin{array}{l}\text { Humans } \\
(n=12)\end{array}$} & Homo sapiens & Human & $10(52.6)$ & $0(0.0)$ & $2(10.5)$ & $12(10.5)$ \\
\hline & & Total & $12(63.2)$ & $3(15.8)$ & $4(21.1)$ & $19(100.0)$ \\
\hline
\end{tabular}

Blood-fed Cx. pipiens and Cs. annulata were captured in sufficient numbers for a more detailed host preference analysis. Significant differences were found in Chester Zoo in 2017 (Fisher's exact test $P<0.001$ ) where $C x$. pipiens preferred to feed on humans and birds, and Cs. annulata preferred to feed on humans and non-human mammals. In the following year, $C x$. pipiens also preferred birds and humans and $C s$. annulata preferred non-human mammals (Fisher's exact test $P=0.025$ ). In 2019, the number of samples per group was not sufficient for statistical analysis as most of the $C x$. pipiens were feeding on birds $(n=17)$. The difference in host preference was also significant in Flamingo Land (Fisher's exact test $P=0.021)$, with $C x$. pipiens preferring humans and Cs. annulata, non-human mammals. Analysing differences in host preferences by month, we found a significant difference in Chester Zoo in 2017 (Fisher's exact test $\mathrm{P}=0.003$ ), Cx. pipiens preferred to feed on humans during June and on birds during July (Fig. 6). Data was insufficient for other comparisons, or differences were not significant.

\section{Distribution of feeding activity}

We observed significant differences in blood-fed mosquito catches among sampling areas in all our samplings. In Chester Zoo, during the first year, areas A1 and A3 captured the largest numbers of mosquitoes and highest proportions of blood-feds; areas A7 and A10 also captured more blood-fed mosquitoes than expected randomly but not in relation to a large mosquito catch $\left(X^{2}=17.556, d f=9, P=0.041\right)$. The following year, area $A 10$ captured more blood-fed mosquitoes, along with areas $A 1$ and $A 11\left(X^{2}=17.894, d f=7, P=0.0125\right)$. In the last year, areas $A 12, A 13$ and $A 3$ yielded high proportions of blood-fed mosquitoes and in this occasion, captures in area $A 1$ were less than expected by chance $\left(X^{2}=16.55, d f=4, P=0.002\right)(F i g .7 a)$. We also found a significant difference in Flamingo Land related to a large catch of blood-fed mosquitoes in area $\mathrm{A} 2$ and smaller catches in areas $\mathrm{A} 3$ and $\mathrm{A} 4$ ( $\mathrm{X}^{2}=$ 24.868, df =3, $P<0.0001$ ) (Fig. 8a).

\section{Seasonality of feeding activity}


The proportion of blood fed mosquitoes trapped also changed between months. A significantly higher proportion of blood-fed mosquitoes in June and May and a lower proportion in July, were observed in Chester Zoo in the first year of sampling $\left(X^{2}=50.596, d f=6, P<0.001\right)$. In 2018, catches larger than expected randomly were observed in July and August and smaller than expected in September and October $\left(X^{2}=54.346, d f=6, P<0.0001\right)$. The sampling in 2019 captured more blood-fed mosquitoes in July and May and less in June $\left(X^{2}=7.15, d f=2, P=0.028\right)$ (Fig. 7b). Likewise, in Flamingo Land a higher proportion of blood-fed mosquitoes was observed in July and August compared to other months $\left(X^{2}=106.51, d f=5, P<0.0001\right)($ Fig. 8b).

\section{Travelling distances}

We identified 14 zoo host species from mosquito blood-meals in Chester Zoo in 2017, five in 2018, three in 2019, and five in Flamingo Land. The minimum travelling distance observed overall was $13.72 \mathrm{~m}$ and the maximum, $366.74 \mathrm{~m}$ (Table 6 ). By species, the average minimum travelling distance was $122.5 \mathrm{~m}$ for $C x$. pipiens (median $=113.3 \mathrm{~m}$ ), $131.8 \mathrm{~m}$ for $C$ s. annulata (median $=56.2 \mathrm{~m}$ ) and $20.3 \mathrm{~m}$ for An. maculipennis s.l. (median $=20.3 \mathrm{~m}$ )

(Figs. 1, 2). A comparison by species (excluding An. maculipennis s.l. due to low numbers) showed no significant difference overall (Kruskal-Wallis, $\mathrm{X}^{2}=$ $0.279, \mathrm{df}=1, \mathrm{P}=0.597)$. One third of the mosquitoes that fed on zoo animals $(\mathrm{n}=9)$ were captured within $50 \mathrm{~m}$ from the exhibits where they had fed on and more than half $(n=15)$ within $100 \mathrm{~m}$. 
Table 6

Minimum travelling distances of blood-fed mosquitoes that fed on zoo animals.

\begin{tabular}{|c|c|c|c|c|c|}
\hline \multirow{2}{*}{$\begin{array}{l}\text { Sampling } \\
\text { Season }\end{array}$} & \multirow[t]{2}{*}{ Mosquito } & \multicolumn{2}{|l|}{ Zoo animal } & \multirow{2}{*}{$\begin{array}{l}\text { Sampling } \\
\text { Area }\end{array}$} & \multirow{2}{*}{$\begin{array}{l}\text { Minimum flying } \\
\text { distance }(m)\end{array}$} \\
\hline & & Scientific name & Common name & & \\
\hline \multirow{14}{*}{$\begin{array}{l}\text { Chester Zoo } \\
2017\end{array}$} & \multirow[t]{7}{*}{ Cx. pipiens } & Camelus bactrianus & Bactrian camel & A1 & 234.3 \\
\hline & & Cissa thalassina & Javan Green Magpie & A4 & 58 \\
\hline & & Cissa thalassina & Javan Green Magpie & A3 & 34.16 \\
\hline & & $\begin{array}{l}\text { Giraffa camelopardalis rothschildi / } \\
\text { Tauraco schalowi }\end{array}$ & $\begin{array}{l}\text { Rothschild's giraffe / } \\
\text { Schalow's turaco }\end{array}$ & A1 & $303.28^{a}$ \\
\hline & & Tauraco schalowi & Schalow's turaco & A1 & 168.58 \\
\hline & & Tauraco schalowi & Schalow's turaco & A1 & 168.58 \\
\hline & & Tauraco schalowi & Schalow's turaco & A7 & 204.7 \\
\hline & \multirow[t]{6}{*}{ Culiseta spp. } & Camelus bactrianus & Bactrian camel & A7 & 56.18 \\
\hline & & Camelus bactrianus & Bactrian camel & A7 & 56.18 \\
\hline & & Camelus bactrianus & Bactrian camel & A7 & 56.18 \\
\hline & & Rucervus eldii thamin & Eld's deer & A1 & 336.73 \\
\hline & & Rucervus eldii thamin & Eld's deer & A11 & 55.88 \\
\hline & & Tragelaphus eurycerus & Bongo & A10 & 107.06 \\
\hline & Unknown* & Tauraco schalowi & Schalow's turaco & A1 & 156.61 \\
\hline \multirow[t]{5}{*}{$\begin{array}{l}\text { Chester Zoo } \\
2018\end{array}$} & $\begin{array}{l}\text { An. } \\
\text { maculipennis }\end{array}$ & Spheniscus humboldti & Humboldt penguin & $\mathrm{A} 2$ & 21.34 \\
\hline & Cx. pipiens & Strix leptogrammica & Brown Wood-Owl & A13 & 185.26 \\
\hline & \multirow[t]{2}{*}{ Culiseta spp. } & Camelus bactrianus & Bactrian camel & A11 & 178.07 \\
\hline & & Rucervus eldii thamin & Eld's deer & $\mathrm{A} 11$ & 55.88 \\
\hline & Unknown* & Spheniscus humboldti & Humboldt penguin & A2 & 21.34 \\
\hline \multirow{3}{*}{$\begin{array}{l}\text { Chester Zoo } \\
2019\end{array}$} & Cx. pipiens & Phoenicopterus ruber & Caribbean flamingo & A1 & 28.41 \\
\hline & Cx. pipiens & Spheniscus humboldti & Humboldt penguin & A12 & 13.72 \\
\hline & $\begin{array}{l}\text { An. } \\
\text { maculipennis }\end{array}$ & Spheniscus humboldti & Humboldt penguin & A12 & 19.19 \\
\hline \multirow{5}{*}{$\begin{array}{l}\text { Flamingo } \\
\text { Land }\end{array}$} & Cx. pipiens & Hydrochoerus hydrochaeris & Capybara & A2 & 46.67 \\
\hline & \multirow[t]{3}{*}{ Culiseta spp. } & Camelus bactrianus & Bactrian camel & A4 & 24.76 \\
\hline & & Hydrochoerus hydrochaeris & Capybara & A1 & 49.23 \\
\hline & & Oryx dammah & Scimitar-horned Oryx & A1 & 366.74 \\
\hline & Unknown* & Oryx dammah & Scimitar-horned Oryx & A2 & 292.94 \\
\hline
\end{tabular}

a: Mixed blood-meals, includes the distance between the exhibits of both animals and the trap. * These mosquitoes were only identified to the Culicinae subfamily due to damaged legs. Sampling areas are presented in Fig. 2 for Chester Zoo and Fig. 3 for Flamingo Land.

\section{Environmental factors}

Six variables showed significant influence on blood-fed mosquito captures, although the direction of influence was not consistent. Temperature had a positive influence in blood-fed mosquito captures in four models (close to significance in one, $\mathrm{P}=0.073$ ) and a negative influence in another, humidity had a negative influence in three models and a positive influence in one, precipitation was a positive influence in three models, wind speed was positively influencing in one model and negatively in another, and scarce vegetation and close distance to zoo animal exhibits had a positive influence in one model. A summary of estimates and $P$ values can be found in supplemental material Table S3.

\section{Discussion}

We confirmed the intrinsic preference of Culex pipiens for birds, mainly free wild birds and some birds from the zoos' collections, and of Culiseta annulata for mammals. Cx. pipiens is primarily an ornithophilic species $[35,40]$, however we observed high proportions of human hosts in all our samplings, except for 2019; similarly Heym et al. [3] and Börstler et al. [41] found mosquitoes feeding on humans but the proportion we observed was higher overall. We 
discount the possibility of major sample contamination as negative controls did not produce a positive result and no sequenced samples matched the positive control. These mosquitoes fed on humans from April to August, when the zoos have more visitors and temporary staff, and no human bloodmeals were found at other times, although the occurrence of birds and other mammalian blood-meals continued to be observed. Additionally, the preference for humans was significantly higher in Cx. pipiens than in Cs. annulata, which is unexpected as $C$ s. annulata has been reported as a biting nuisance for people in the UK [42]. PCR identification showed that all Cx. pipiens that fed on humans belong to the pipiens biotype; as this biotype is typically described as ornithophilic, abundance of visitors and staff seem to be a relevant influence in mosquito feeding preferences in zoos.

Anopheles maculipennis s.l. prefers to feed on mammals than on birds [32, 43]. However, two mosquitoes from this group fed on Humboldt penguins (Spheniscus humboldti), which to our knowledge is the first report of this host choice. Due to the low sample size, we cannot conclude if there is a host preference or simply a tendency of capturing blood-fed mosquitoes in proximity to the animals they feed on as these mosquitoes were captured close to the penguin exhibit $(<22 \mathrm{~m})$. Therefore, targeted sampling of this group is needed and should include the molecular identification of the species as they have different host preferences [43].

A correlation between mosquito abundance and proportion of blood-feds has been noticed before [21]. During the first year in Chester Zoo, we found high mosquito abundance and high proportion of blood-feds in areas A1 and A3 and again for A1 in 2018. In Flamingo Land, we observed a similar situation in area A2. Nevertheless, other areas with lower general catches showed high proportions of blood-fed mosquitoes, like A7 and A10 in Chester Zoo in 2017 and areas A10 and A11 in 2018. Therefore, the mosquito abundance is not the only explanatory factor. For instance, area A3 in Chester Zoo captured a high number of blood-fed mosquitoes in 2017 possibly due to its proximity to a picnic area and a children's playground. Fewer mosquitoes were caught in this area in 2018, when the children's playground was closed for renewal over several weeks, and the off-show aviaries next to the sampling area were expanded, reducing a considerable portion of the vegetation. In Chester Zoo, area A7, which caught more blood-feds than expected in 2017, is also near a picnic garden and an area with high transit of visitors. In 2017 and 2018, area A10 also captured a high proportion of blood-feds which matched mainly wild birds. This area is inside a wetland aviary where several species are kept, and the abundance of wild passerines could be high as they are attracted to the waterfowl's food despite the netting of the exhibit. In Flamingo Land, area A2, also with a higher proportion of blood-feds, is in the boundary of the South American exhibit which contains large mammals such as capybara (Hydrochoerus hydrochaeris), on which we found two mosquitoes feeding. It appears from this result that the constant presence of suitable hosts is an attractant factor for mosquitoes.

The mosquito host feeding preferences varied each year. There is evidence that host selection by Culex spp. is influenced primarily by the availability of preferred hosts [44]; therefore, these variations possibly depend on the mosquito abundance and host availability, both of which increase during the summer. We observed a significant host shift in the case of CX. pipiens in Chester Zoo in 2017, preferring humans in June and birds in July. Tuten et al. [2] also observed a host shift for $C x$. pipiens, although in this case preferring birds in the summer and birds and mammals in autumn. These changes could be related to host availability influenced for instance, by the migration and breeding seasons of birds (nestlings are more prone to mosquito bites [3]). Furthermore, when the preferred hosts of Culex spp. are scarce, this mosquito can shift to other hosts, including humans [12].

Some species like $C x$. pipiens breed and rest close to their host's habitat [20], so it is reasonable that the chances of capturing blood-fed mosquitoes are higher closer to the location of potential hosts as we observed for zoo animals. Other authors reported maximum travelling distances between 170 to $770 \mathrm{~m}[2-4,22]$ and the maximum that we observed was within this range (367 m). However, we found mosquitoes feeding on domestic animals (cattle, pig, chicken, and dog) that we assume came from nearby farms. Thus, dispersal was probably further than our estimate.

It is possible that in some areas the landscape forms flight paths that aids mosquito's movement in a certain direction [22] and the dominant wind direction could be a relevant factor influencing dispersal and flight direction [45]. For example, Area A1 in Chester Zoo captured blood-fed mosquitoes that feed on zoo animals located from the southwest to the northwest from this area, and the wind on the day before collection came from a similar direction in the case of four out of six mosquitoes. The use of portable weather stations could improve the study of wind and landscape influence in mosquito dispersal. It is important to consider that using the exhibit's centroid, or any other measurement to the exhibit, assumes that the animals distribute randomly within their exhibits, when in reality, animals spend more time in certain areas, like around the feeders during the day or in the enclosure at nighttime. The estimation of animal occupancy degrees inside the exhibits should be considered for a more precise assessment of mosquito travelling distances.

Temperature affects mosquito feeding activity, reproduction, longevity, and development [46, 47], which could explain the positive relationship with the capture of blood-fed mosquitoes showed in the models. High relative humidity may enhance the effect of odorant cues for host-seeking mosquitoes [12]; however, our models showed mostly a negative relation, possibly because higher humidity promotes mosquito dispersal [20] and thus decreases the chances of capture. Precipitation on the other hand, significantly increased the chances of capturing blood-fed mosquitoes according to three models; this is counterintuitive as mosquitoes are not expected to fly under rainy conditions. However, this variable was aggregated weekly over the sampling units; thus, higher values do not imply more constant rain but more rain over a week period. Therefore, rainfall may reduce mosquito dispersal increasing the capture chances but does not prevent their feeding activity. Both, Brugman et al. [32] and Karki et al., [45] reported that the wind diminishes the capture of blood-fed mosquitoes, which can be explained by mosquitoes taking shelter under windy conditions, thus reducing the host-seeking activity and the dispersal after blood-feeding. Wind speed was significant in two models but in opposite directions, so we cannot conclude its influence in our samplings. In one model, scarce vegetation was associated with an increase in blood-fed mosquitoes capture suggesting that mosquitoes that are looking for shelter are attracted to the traps in absence of natural resting areas. Finally, a close distance to zoo animal exhibits significantly increased the likelihood of capturing blood-fed mosquitoes in one model, possibly due to dispersal behaviour as discussed before in relation to travelling distances. 
After blood feeding, mosquitoes look for a resting place to digest the blood and produce eggs; thus blood-engorged females tend to be captured in low proportions in mosquito traps that target host seeking individuals (e.g. [26]) or gravid females looking to oviposit, which varies depending on the sampling methods and study sites (e.g. [2-4, 22]). It is not clear if blood-fed mosquitoes were attracted by the lactic acid of host-seeking mosquito traps because they are looking for a second blood-meal, or by the fermenting media of gravid traps because they prefer to rest near potential oviposition sites. Alternatively, they may be attracted by the dark colour and location of the traps. However, we cannot exclude the possibility that they were randomly captured as no significant differences were found between BG-Mosquitaire and CDC-Gravid trap types. We captured higher proportions of blood-fed mosquitoes using resting traps and aspirating resting areas, as reported by other authors $[2,32,48]$. In some cases, the use of resting traps has failed [2, 49], thus their positioning and orientation could be a determinant and should ensure that mosquitoes get protection from direct sunlight, rain, and wind.

Our blood-meal identification success could have been influenced by the time before collecting the mosquitoes and weather conditions. If the mosquitoes remained alive, they continued digesting the blood, and if dead, they started to desiccate. We observed more mosquitoes completely dehydrated, despite their evident blood-fed status, during the 2018 sampling in Chester Zoo which was drier and hotter compared to other sampling years. To improve the results, storing the samples at $-80^{\circ} \mathrm{C}$ or in filter paper and processing them promptly has been recommended [29, 31, 32], which would also minimise physical damage facilitating mosquito morphological identification.

The high proportion of $C x$. pipiens mosquitoes feeding on humans that we observed represents not only a likely nuisance for visitors and staff at the zoos, but also a potential risk for disease transmission. Culex spp. are vectors of viruses hosted by wild birds, such as West Nile virus (WNV), Sindbis virus (SINV) and Usutu virus (USUV), all of which have been reported circulating in mainland Europe $[17,50]$ and could pose a serious threat if they are introduced to the UK. Moreover, all mixed blood-meals that we found were from $C x$. pipiens involving a bird host and the mixed blood meals that included humans were combined with blood of Eurasian magpie (Pica pica), which is a proven natural reservoir of WNV and an effective target for its surveillance [51]. In addition, it has been shown that the temporal and spatial variation in host preferences by Culex spp. could influence the timing and severity of WNV infections, probably in relation to its seasonal shifts between ornithophilic and anthropophilic cycles [7, 21]. Although the host shift in Cx. pipiens that we observed in one of our samplings occurred from humans to birds, the potential of this mosquito as a bridge vector for humans and domestic animals (i.e. horses) $[49,52]$ should be constantly monitored, despite the lack of evidence confirming WNV's establishment in the UK [17, 53], as well as for the other mentioned viruses (SINV and USUV).

The interspecific transmission of vector borne diseases is also important for the health of the animals in the zoo collections. For instance, mosquitoes have been involved in the transmission of Eastern equine encephalitis virus to African penguins (Spheniscus demersus) in North America, USUV to great grey owls (Strix nebulosa) in Austria and WNV that has caused the death of exotic animals in roughly one hundred zoos in the United States [1]. The risk of disease transmission between local bird species and zoo animals is present in our study sites, as avian malaria has caused outbreaks in Humboldt penguin (Spheniscus humboldti) colonies in both Chester Zoo and Flamingo Land [10]. Interestingly, host preference changes could also influence the transmission dynamics of avian malaria parasites in bird communities [44]. We found four mosquitoes feeding on Humboldt penguins, two Anopheles maculipennis s. I., one $C x$. pipiens and an unknown Culicinae, which is likely to be $C x$. pipiens as it had all the corresponding morphological features except for those evaluated on the legs. Anopheles spp. mosquitoes are considered as potential vectors for avian Plasmodium spp. and have been found susceptible to the parasite infection experimentally [54]. Therefore, this genus could have a relevant role in the transmission of avian malaria, although An. maculipennis s. I. has not been found infected with avian Plasmodium yet [55,56]. To clarify the host preferences of this mosquito genus and Plasmodium spp. transmission risks, the precise identification of its species is needed.

\section{Conclusions}

Zoological gardens provide unique opportunities for the study of mosquitoes; thus, abundance, host choice and dispersal can be explored to assess disease transmission risks. We confirmed that mosquitoes in zoos conserve their characteristic host preferences up to certain degree, feed on a wide range of hosts, and presented an important and unexpected preference for humans which varied between 10 and $63 \%$ in our samplings. This highlights the risk of zoonotic viruses transmitted between humans and birds such as WNV, USUV and SINV if ever stablished in the UK. There is an implied health risk for zoo birds as we found mosquitoes feeding on them. Of especial concern are the Javan green magpies (Cissa thalassina) which are critically endangered, and the Humboldt penguins (Spheniscus humboldti) due to their high susceptibility to avian malaria. Mosquito feeding behaviour is influenced by different factors and it changes temporally and spatially. In our samplings, the main period of feeding activity varied with year and location, it corresponded with the overall increase in mosquito abundance and it was mainly influenced by temperature in a positive sense. We captured a high proportion of blood-fed mosquitoes in areas with high captures of mosquitoes in general, but this was not always the case. Mosquito dispersal after a blood-meal was variable and it is likely that landscape features influence their movements. Therefore, mosquitoes feeding behaviour is influenced by the weather, landscape, and abundance of potential hosts. Our results highlight the complexity of mosquito ecology in zoos and the relevance of assessing interspecific transmission risk of pathogens which need to be thoroughly understood for the efficient control of mosquito populations and reducing vectorhost contact in zoological gardens.

\section{Declarations}

\section{Ethics approval}

This project was approved by the science committees of Chester Zoo and Flamingo Land, and by the University of Liverpool Veterinary Research Ethics Committee (reference VREC532a). 


\section{Consent for publication}

Not applicable

\section{Availability of data and materials}

All data relevant for this study is contained in this published article and the supplementary information files. The datasets for this study are available from the corresponding author on reasonable request.

\section{Competing interests}

The authors declare that they have no competing interests.

\section{Funding}

Chester Zoo and the Houghton Trust partially funded the field and lab work of is study as part of an avian malaria research project in UK zoos. AHC and MGO were supported by CONACYT (National Science and Technology Council, Mexico) to perform their PhD studies from which this work derives, and they were also supported by Chester Zoo as members of the Conservation Scholar and Fellow Program.

\section{Authors' contributions}

Study conceived and designed: AHC, MGO, MB, JL, LE, and DW. Mosquito sampling in Chester Zoo in 2017: AHC, MGO, EL; sampling in 2018: AHC and MGO; sampling in 2019: FT and AHC. Sampling in Flamingo Land (2017): DW, AHC, MGO, and EL. Mosquito identification and blood-meal analysis in 2017: EL and AHC; in 2018: AM, FT and AHC; in 2019: FT and AHC. Sampling and laboratory techniques chosen by AHC, JCH and KS. Data analysis: AHC. Funding acquired: $\mathrm{AHC}, \mathrm{MB}$ and $\mathrm{JL}$. Original manuscript written: $\mathrm{AHC}$ with comments from $\mathrm{MB}, \mathrm{JL}, \mathrm{JCH}, \mathrm{EL}, \mathrm{FT}$, and $\mathrm{AM}$. The final version was approved by all authors.

\section{Acknowledgments}

The authors are grateful for the assistance in Chester Zoo from Andrew Woolham, Mark Vercoe, Andrew Owen, Victoria Davis, Andrea Green, Gabby Drake, Wayne McLeod, and the animal keeper teams, and in Flamingo Land from Ross Snipp. Thanks to Sören Metelmann for providing weather data, Marie Mclntyre for her advice in the model construction, and Julian Chantrey, Rob Christley, Marcus Blagrove and Andrew Jackson for reviewing and making valuable suggestions to the project. The 2019 sampling and lab work were supported by Benjamin Staniforth and Howie Shribman. Finally, the technicians' team in Leahurst campus did all the background work needed for the lab analyses.

\section{References}

1. Adler PH, Tuten HC, Nelder MP. Arthropods of medicoveterinary importance in zoos. Annu Rev Entomol. 2011;56:123-42.

2. Tuten HC, Bridges WC, Paul KS, Adler PH. Blood-feeding ecology of mosquitoes in zoos. Med Vet Entomol. 2012;26:407-16; doi: 10.1111/j.13652915.2012.01012.x.

3. Heym EC, Kampen H, Schafer M, Walther D. Mosquito bloodmeal preferences in two zoological gardens in Germany. Med Vet Entomol. 2019;33:20312; doi: 10.1111/mve.12350.

4. Greenberg JA, DiMenna MA, Hanelt B, Hofkin BV. Analysis of post-blood meal flight distances in mosquitoes utilizing zoo animal blood meals. J Vector Ecol. 2012;37:83-9; doi: 10.1111/j.1948-7134.2012.00203.x.

5. Ludwig GV, Calle PP, Mangiafico JA, Raphael BL, Danner DK, Hile JA, et al. An outbreak of West Nile virus in a New York City captive wildlife population. Am J Trop Med Hyg. 2002;67:67-75; doi: 10.4269/ajtmh.2002.67.67.

6. Lanciotti RS, Roehrig JT, Deubel V, Smith J, Parker M, Steele K, et al. Origin of the West Nile virus responsible for an outbreak of encephalitis in the northeastern United States. Science. 1999;286:2333-7; doi: 10.1126/science.286.5448.2333.

7. Kilpatrick AM, Kramer LD, Jones MJ, Marra PP, Daszak P. West Nile virus epidemics in North America are driven by shifts in mosquito feeding behavior. PLoS Biol. 2006;4:e82; doi: 10.1371/journal.pbio.0040082.

8. Silveira P, Belo NO, Lacorte GA, Kolesnikovas CK, Vanstreels RE, Steindel M, et al. Parasitological and new molecular-phylogenetic characterization of the malaria parasite Plasmodium tejerai in South American penguins. Parasitol Int. 2013;62:165-71; doi: 10.1016/j.parint.2012.12.004.

9. Grilo ML, Vanstreels RE, Wallace R, Garcia-Parraga D, Braga EM, Chitty J, et al. Malaria in penguins: current perceptions. Avian Pathol. 2016;45:393407; doi: 10.1080/03079457.2016.1149145.

10. Hernandez-Colina A. Ecology of mosquito vectors in relation to avian malaria in zoological gardens in the United Kingdom. PhD thesis. Liverpool: The University of Liverpool; 2019. 
11. Stephenson EB, Murphy AK, Jansen CC, Peel AJ, McCallum H. Interpreting mosquito feeding patterns in Australia through an ecological lens: an analysis of blood meal studies. Parasit Vectors. 2019;12:156; doi: 10.1186/s13071-019-3405-z.

12. Takken W, Verhulst NO. Host preferences of blood-feeding mosquitoes. Annu Rev Entomol. 2013;58:433-53; doi: 10.1146/annurev-ento-120811153618.

13. Ciota AT, Kramer LD. Vector-virus interactions and transmission dynamics of West Nile virus. Viruses. 2013;5:3021-47; doi: 10.3390/v5123021.

14. Abella-Medrano CA, Ibanez-Bernal S, Carbo-Ramirez P, Santiago-Alarcon D. Blood-meal preferences and avian malaria detection in mosquitoes (Diptera: Culicidae) captured at different land use types within a neotropical montane cloud forest matrix. Parasitol Int. 2018;67:313-20; doi: 10.1016/j.parint.2018.01.006.

15. Farajollahi A, Fonseca DM, Kramer LD, Marm Kilpatrick A. "Bird biting" mosquitoes and human disease: a review of the role of Culex pipiens complex mosquitoes in epidemiology. Infection, genetics and evolution : Infect Genet Evol. 2011;11:1577-85; doi: 10.1016/j.meegid.2011.08.013.

16. Pinheiro RB, Felix GM, Chaves AV, Lacorte GA, Santos FR, Braga EM, et al. Trade-offs and resource breadth processes as drivers of performance and specificity in a host-parasite system: a new integrative hypothesis. Int J Parasitol. 2016;46:115-21; doi: 10.1016/j.ijpara.2015.10.002.

17. Folly A, Dorey-Robinson D, Hernández-Triana L, Phipps $L$, Johnson N. Emerging threats to animals in the United Kingdom by arthropod-borne diseases. Front Vet Sci. 2020;7:20; doi: 10.3389/fvets.2020.00020.

18. Schonenberger AC, Wagner S, Tuten HC, Schaffner F, Torgerson P, Furrer S, et al. Host preferences in host-seeking and blood-fed mosquitoes in Switzerland. Med Vet Entomol. 2016;30:39-52; doi: 10.1111/mve.12155.

19. Faust CL, Dobson AP, Gottdenker N, Bloomfield LSP, McCallum HI, Gillespie TR, et al. Null expectations for disease dynamics in shrinking habitat: dilution or amplification? Philos Trans R Soc Lond B Biol Sci. 2017;372:20160173; doi: 10.1098/rstb.2016.0173.

20. Becker N, Petric D, Zgomba M, Boase C, Dahl C, Madon M, et al. Mosquitoes and their control. 2nd ed. Berlin: Springer-Verlag Berlin; 2010.

21. Goodman H, Egizi A, Fonseca DM, Leisnham PT, LaDeau SL. Primary blood-hosts of mosquitoes are influenced by social and ecological conditions in a complex urban landscape. Parasit Vectors. 2018;11:218; doi: 10.1186/s13071-018-2779-7.

22. Ejiri H, Sato Y, Kim KS, Hara T, Tsuda Y, Imura T, et al. Entomological study on transmission of avian malaria parasites in a zoological garden in Japan: bloodmeal identification and detection of avian malaria parasite DNA from blood-fed mosquitoes. J Med Entomol. 2011;48:600-7; doi: 10.1603/me10197.

23. Biogents AG. BG-Mosquitaire against tiger mosquitoes. 2017. http://www.biogents.com/bg-mosquitaire/. Accessed 03 Jan 2017.

24. John W. Hock Company. CDC gravid trap. 2013. http://johnwhock.com/products/mosquito-sandfly-traps/cdc-gravid-trap/. Accessed 03 Jan 2017.

25. Reiter P. A portable, battery-powered trap for collecting gravid Culex mosquitos. Mosq News. 1983;43:496-8.

26. Roiz D, Roussel M, Munoz J, Ruiz S, Soriguer R, Figuerola J. Efficacy of mosquito traps for collecting potential West Nile mosquito vectors in a natural Mediterranean wetland. Am J Trop Med. 2012;86:642-8; doi: 10.4269/ajtmh.2012.11-0326.

27. John W. Hock Company. Modified CDC backpack aspirator. 2017. https://www.johnwhock.com/products/aspirators/modified-cdc-backpackaspirator/. Accessed 13 Jan 2017.

28. Cranston P, Ramsdale C, Snow K, White G. Adults, larvae and pupae of British mosquitoes (Culicidae): a key. Sci Publ No. 48. Ambleside: Freshwater Biological Association; 1987.

29. Reeves LE, Holderman CJ, Gillett-Kaufman JL, Kawahara AY, Kaufman PE. Maintenance of host DNA integrity in field-preserved mosquito (Diptera: Culicidae) blood meals for identification by DNA barcoding. Parasit Vectors. 2016;9:503; doi: 10.1186/s13071-016-1791-z.

30. Detinova TS. Age-grouping methods in Diptera of medical importance with special reference to some vectors of malaria. Monogr Ser World Health Organ. 1962;47:13-191.

31. Santos CS, Pie MR, da Rocha TC, Navarro-Silva MA. Molecular identification of blood meals in mosquitoes (Diptera, Culicidae) in urban and forested habitats in southern Brazil. PLoS One. 2019;14:e0212517; doi: 10.1371/journal.pone.0212517.

32. Brugman VA, Hernandez-Triana LM, England ME, Medlock JM, Mertens PP, Logan JG, et al. Blood-feeding patterns of native mosquitoes and insights into their potential role as pathogen vectors in the Thames estuary region of the United Kingdom. Parasit Vectors. 2017;10:163; doi: 10.1186/s13071017-2098-4.

33. Alcaide M, Rico C, Ruiz S, Soriguer R, Munoz J, Figuerola J. Disentangling vector-borne transmission networks: a universal DNA barcoding method to identify vertebrate hosts from arthropod bloodmeals. PLoS One. 2009;4:e7092; doi: 10.1371/journal.pone.0007092.

34. Hesson JC, Lundstrom JO, Halvarsson P, Erixon P, Collado A. A sensitive and reliable restriction enzyme assay to distinguish between the mosquitoes Culex torrentium and Culex pipiens. Med Vet Entomol. 2010;24:142-9; doi: 10.1111/j.1365-2915.2010.00871.x.

35. Martinez-de la Puente J, Ferraguti M, Ruiz S, Roiz D, Soriguer RC, Figuerola J. Culex pipiens forms and urbanization: effects on blood feeding sources and transmission of avian Plasmodium. Malar J. 2016;15:589; doi: 10.1186/s12936-016-1643-5.

36. Becker N, Jost A, Weitzel T. The Culex pipiens complex in Europe. J Am Mosq Control Assoc. 2012;28 Suppl 4:53-67; doi: 10.2987/8756-971X28.4s.53.

37. Bahnck CM, Fonseca DM. Rapid assay to identify the two genetic forms of Culex (Culex) pipiens L. (Diptera: Culicidae) and hybrid populations. Am J Trop Med Hyg. 2006;75:251-5.

38. Species360. Zoological Information Management Software (ZIMS). 2019. https://www.species360.org/products-services/zoo-aquarium-animalmanagement-software/. Accessed 13 Aug 2019. 
39. R Core Team. R: a language and environment for statistical computing. 3.0.2 ed. Vienna, Austria: R Foundation for Statistical Computing; 2012.

40. Hesson JC, Schafer M, Lundstrom JO. First report on human-biting Culex pipiens in Sweden. Parasit Vectors. 2016;9:632; doi: 10.1186/s13071-0161925-3.

41. Börstler J, Jöst H, Garms R, Krüger A, Tannich E, Becker N, et al. Host-feeding patterns of mosquito species in Germany. Parasit Vectors. 2016;9:318; doi: 10.1186/s13071-016-1597-z.

42. Asgharian H, Chang PL, Lysenkov S, Scobeyeva VA, Reisen WK, Nuzhdin SV. Evolutionary genomics of Culex pipiens: global and local adaptations associated with climate, life-history traits and anthropogenic factors. Proc Biol Sci. 2015;282: 20150728; doi: 10.1098/rspb.2015.0728.

43. Danabalan R, Monaghan MT, Ponsonby DJ, Linton YM. Occurrence and host preferences of Anopheles maculipennis group mosquitoes in England and Wales. Med Vet Entomol. 2014;28:169-78; doi: 10.1111/mve.12023.

44. Kim KS, Tsuda Y. Seasonal changes in the feeding pattern of Culex pipiens pallens govern the transmission dynamics of multiple lineages of avian malaria parasites in Japanese wild bird community. Mol Ecol. 2010;19:5545-54; doi: 10.1111/j.1365-294X.2010.04897.x.

45. Karki S, Hamer GL, Anderson TK, Goldberg TL, Kitron UD, Krebs BL, et al. Effect of trapping methods, weather, and landscape on estimates of the Culex vector mosquito abundance. Environ Health Insights. 2016;10:93-103; doi: 10.4137/EHI.S33384.

46. Ciota AT, Matacchiero AC, Kilpatrick AM, Kramer LD. The effect of temperature on life history traits of Culex mosquitoes. J Med Entomol. 2014;51:5562; doi: 10.1603/me13003.

47. Ewing DA, Cobbold CA, Purse BV, Nunn MA, White SM. Modelling the effect of temperature on the seasonal population dynamics of temperate mosquitoes. J Theor Biol. 2016;400:65-79; doi: 10.1016/j.jtbi.2016.04.008.

48. Egizi A, Martinsen ES, Vuong H, Zimmerman KI, Faraji A, Fonseca DM. Using bloodmeal analysis to assess disease risk to wildlife at the new northern limit of a mosquito species. EcoHealth. 2018;15:543-54; doi: 10.1007/s10393-018-1371-0.

49. Chapman GE, Archer D, Torr S, Solomon T, Baylis M. Potential vectors of equine arboviruses in the UK. Vet Rec. 2017;180:19; doi: 10.1136/vr.103825.

50. Hesson JC, Verner-Carlsson J, Larsson A, Ahmed R, Lundkvist A, Lundstrom JO. Culex torrentium mosquito role as major enzootic vector defined by rate of Sindbis virus infection, Sweden, 2009. Emerg Infect Dis. 2015;21:875-8; doi: 10.3201/eid2105.141577.

51. Napp S, Montalvo T, Piñol-Baena C, Gómez-Martín MB, Nicolás-Francisco O, Soler M, et al. Usefulness of Eurasian magpies (Pica pica) for West Nile virus surveillance in non-endemic and endemic situations. Viruses. 2019;11:716; doi: 10.3390/v11080716.

52. Hamer GL, Kitron UD, Brawn JD, Loss SR, Ruiz MO, Goldberg TL, et al. Culex pipiens (Diptera: Culicidae): a bridge vector of West Nile virus to humans. J Med Entomol. 2008;45:125-8; doi: 10.1603/0022-2585(2008)45[125:cpdcab]2.0.co;2.

53. Vaux AG, Gibson G, Hernandez-Triana LM, Cheke RA, McCracken F, Jeffries CL, et al. Enhanced West Nile virus surveillance in the North Kent marshes, UK. Parasit Vectors. 2015;8:91; doi: 10.1186/s13071-015-0705-9.

54. Huff CG. Susceptibility of mosquitoes to avian malaria. Exp Parasitol. 1965;16:107-32; doi: 10.1016/0014-4894(65)90036-6.

55. Martinez-de la Puente J, Munoz J, Capelli G, Montarsi F, Soriguer R, Arnoldi D, et al. Avian malaria parasites in the last supper: identifying encounters between parasites and the invasive Asian mosquito tiger and native mosquito species in Italy. Malar J. 2015;14:32; doi: 10.1186/s12936-015-0571-0.

56. Inci A, Yildirim A, Njabo KY, Duzlu O, Biskin Z, Ciloglu A. Detection and molecular characterization of avian Plasmodium from mosquitoes in central Turkey. Vet Parasitol. 2012;188:179-84; doi: 10.1016/j.vetpar.2012.02.012.

\section{Figures}




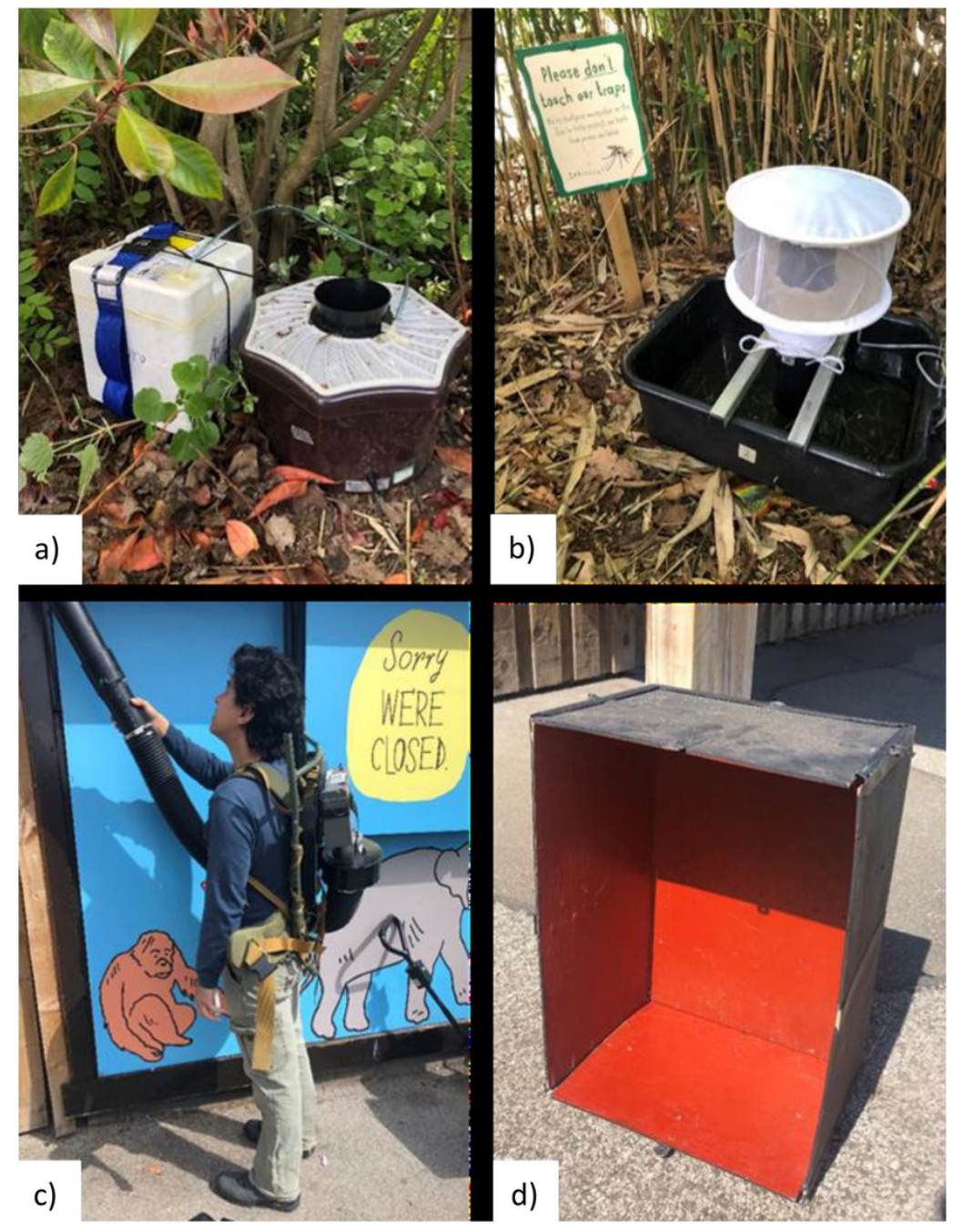

Figure 1

Trapping methods used for mosquito sampling. a) BG-Mosquitaire trap (upgraded with dry ice for the 2019 season); b) CDC-Gravid trap model 1712; c) CDC-Backpack aspirator model 1412 (only used in 2019); d) Resting box trap (only used in 2019). Pictures by FT. 


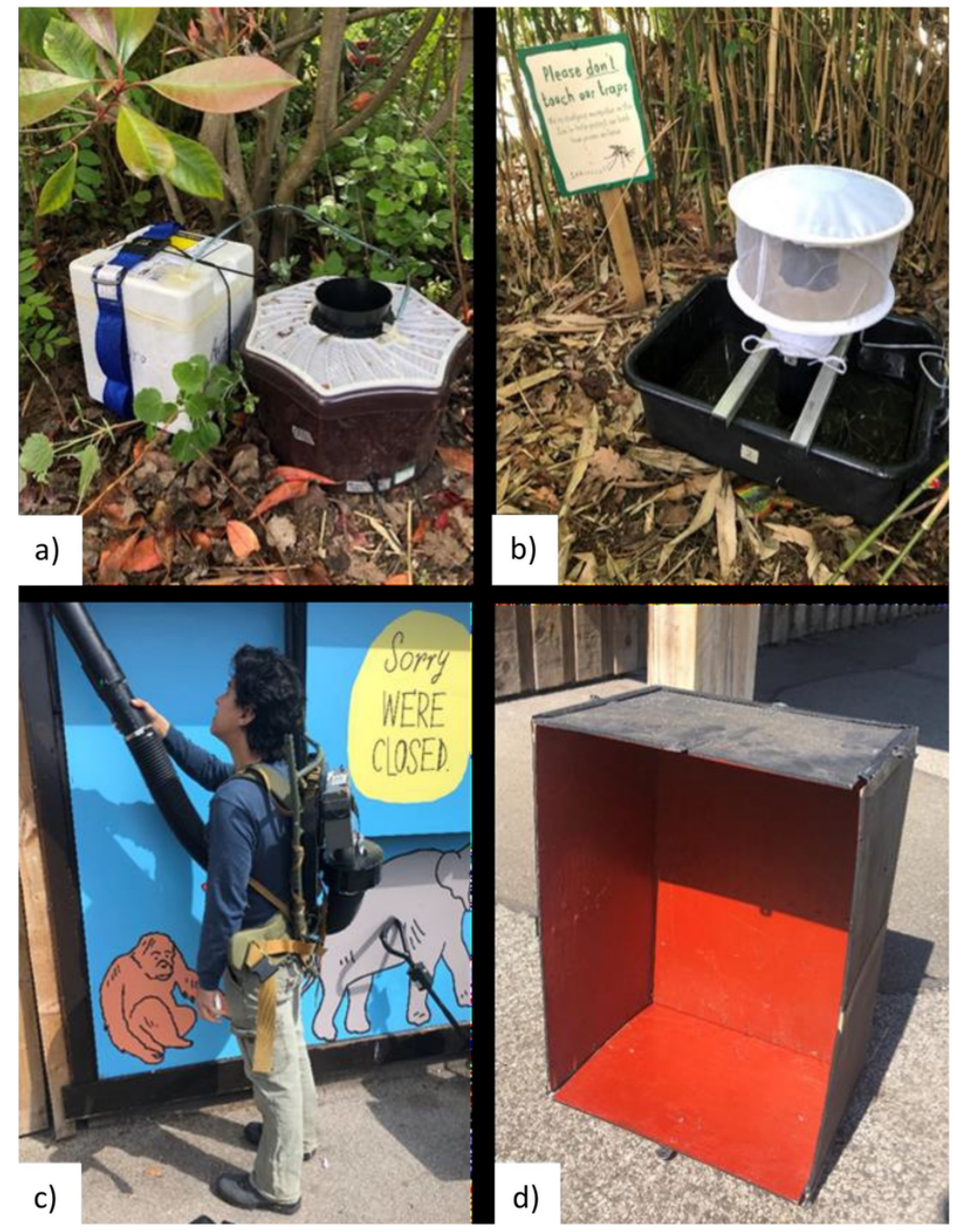

Figure 1

Trapping methods used for mosquito sampling. a) BG-Mosquitaire trap (upgraded with dry ice for the 2019 season); b) CDC-Gravid trap model 1712; c) CDC-Backpack aspirator model 1412 (only used in 2019); d) Resting box trap (only used in 2019). Pictures by FT. 


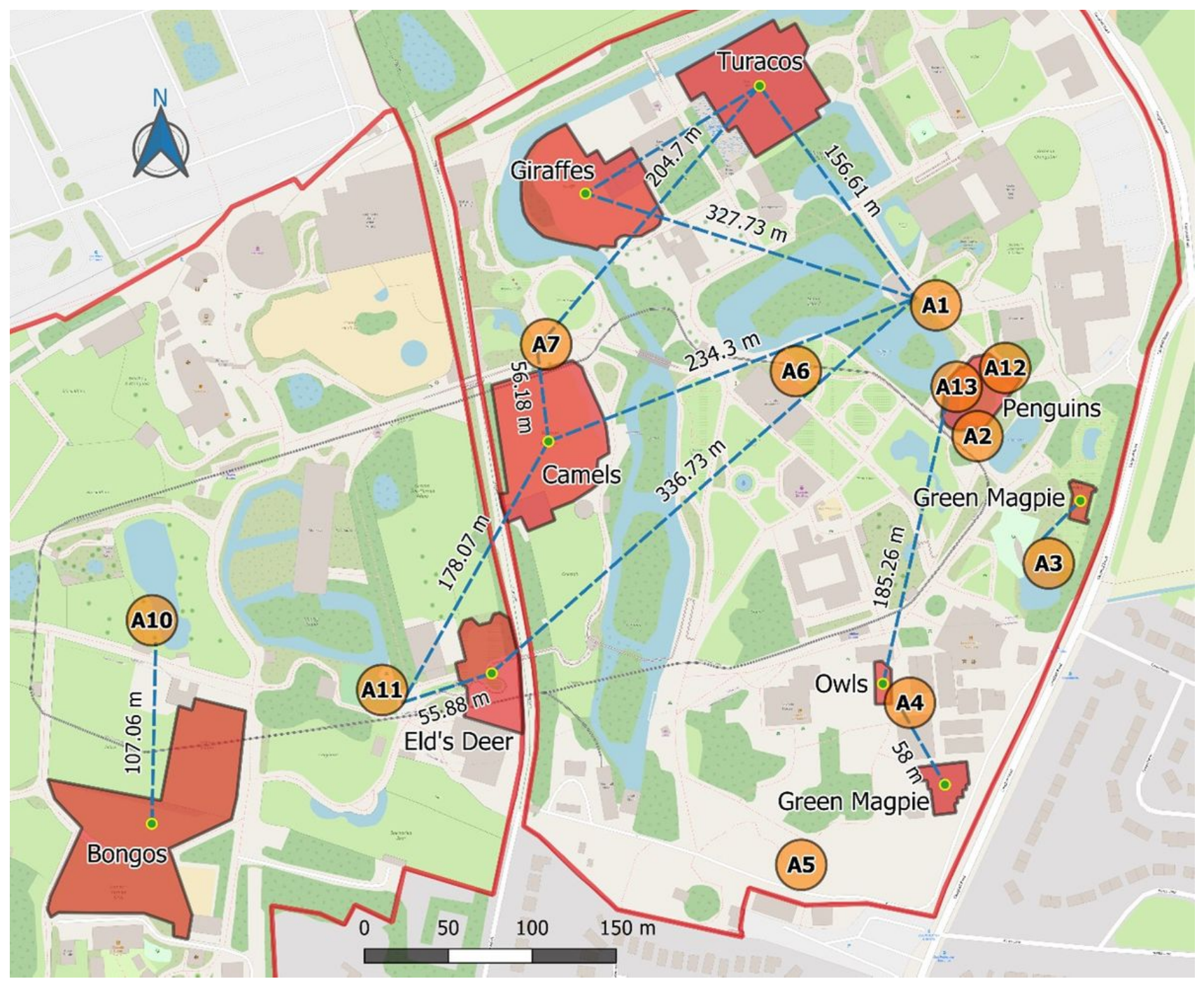

\section{Figure 2}

Sampling areas and minimum travelling distances of blood-fed mosquitoes in Chester Zoo. Not all distances are shown. Red areas: zoo animal exhibits; orange circles: sampling areas; green dots: exhibit centroids; dotted lines: minimum travelling distances; red line: zoo's perimeter. Area A13 was not active in the 2017 sampling; areas A5, A6 and A7 were not active in the 2018 sampling; areas A4, A5, A6, A7, A10 and A11 were not active in the 2019 sampling. 


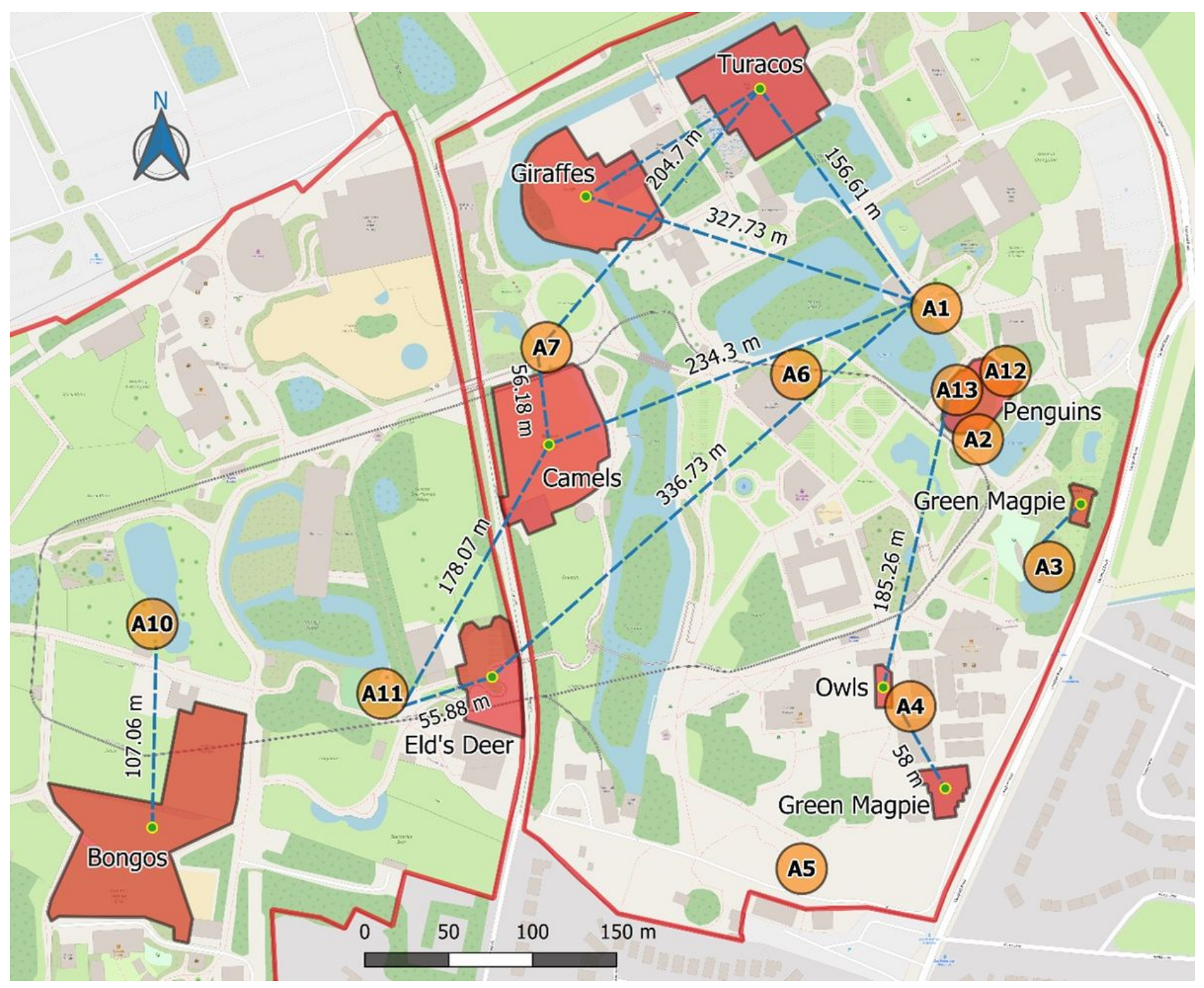

\section{Figure 2}

Sampling areas and minimum travelling distances of blood-fed mosquitoes in Chester Zoo. Not all distances are shown. Red areas: zoo animal exhibits; orange circles: sampling areas; green dots: exhibit centroids; dotted lines: minimum travelling distances; red line: zoo's perimeter. Area A13 was not active in the 2017 sampling; areas A5, A6 and A7 were not active in the 2018 sampling; areas A4, A5, A6, A7, A10 and A11 were not active in the 2019 sampling. 


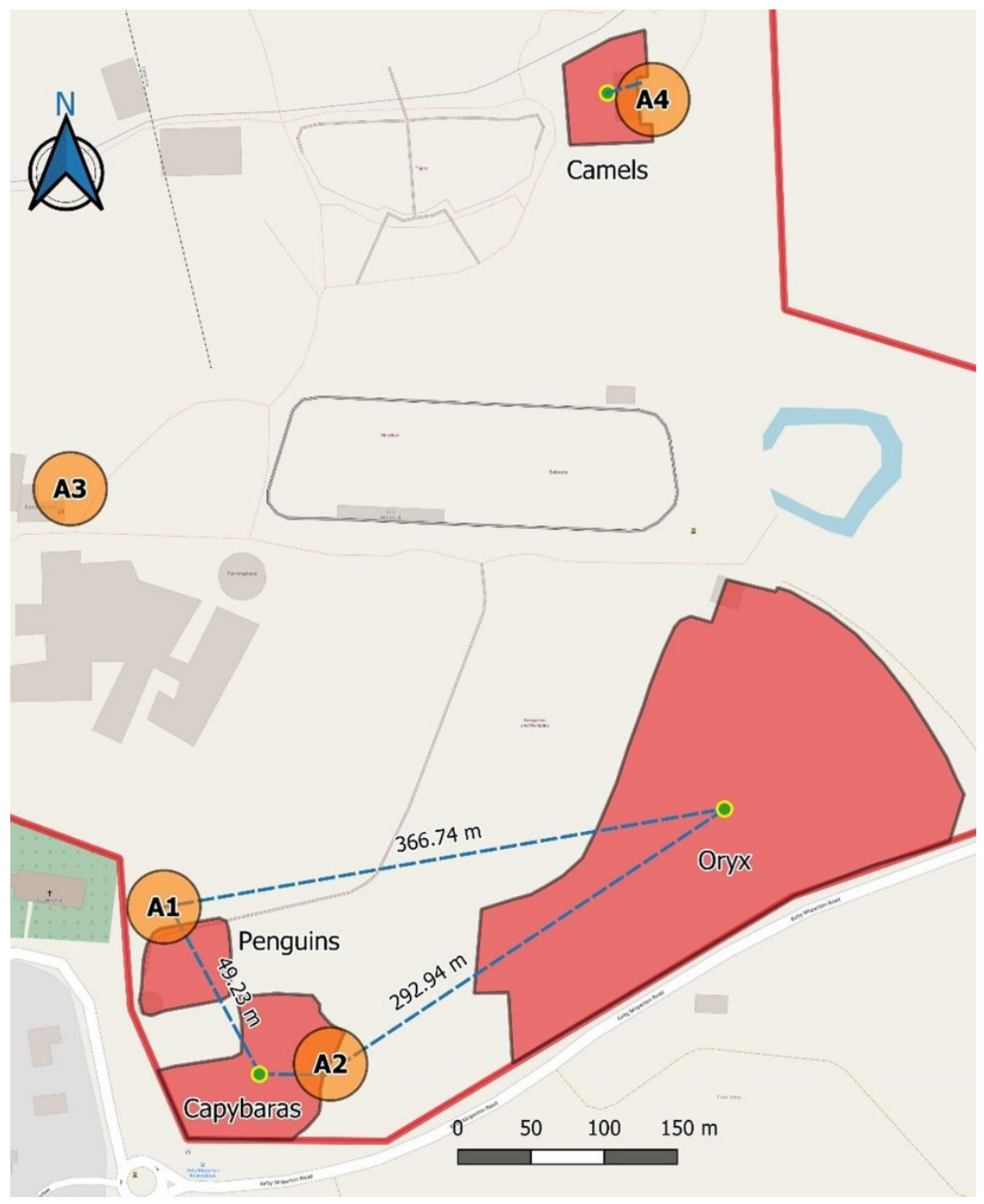

Figure 3

Sampling areas and minimum travelling distances of blood-fed mosquitoes in Flamingo Land, 2017. Not all distances are shown. Red areas: zoo animal exhibits; orange circles: sampling areas; green dots: exhibit centroids; dotted lines: minimum travelling distances; red line: zoo's perimeter. 


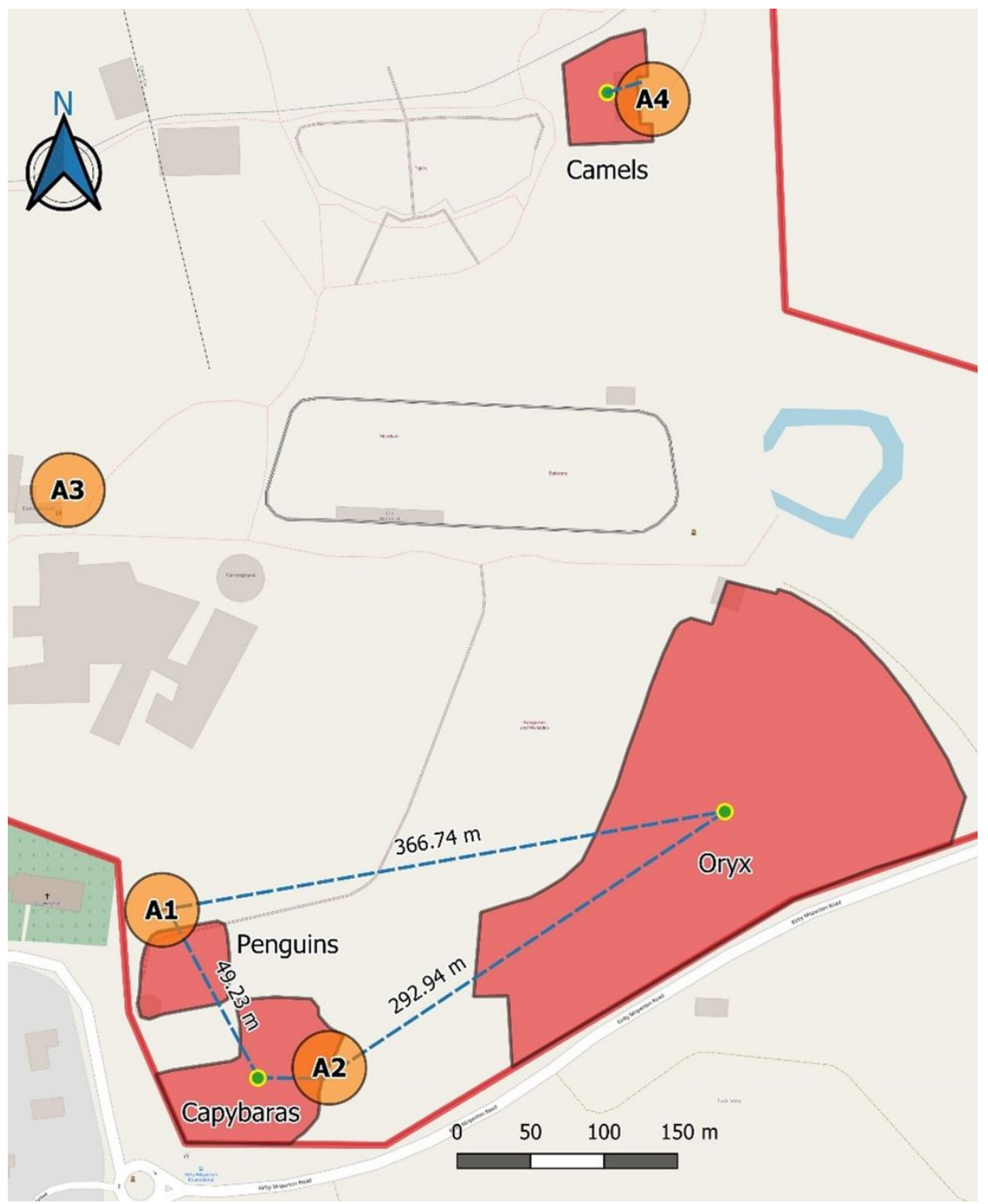

\section{Figure 3}

Sampling areas and minimum travelling distances of blood-fed mosquitoes in Flamingo Land, 2017. Not all distances are shown. Red areas: zoo animal exhibits; orange circles: sampling areas; green dots: exhibit centroids; dotted lines: minimum travelling distances; red line: zoo's perimeter. 


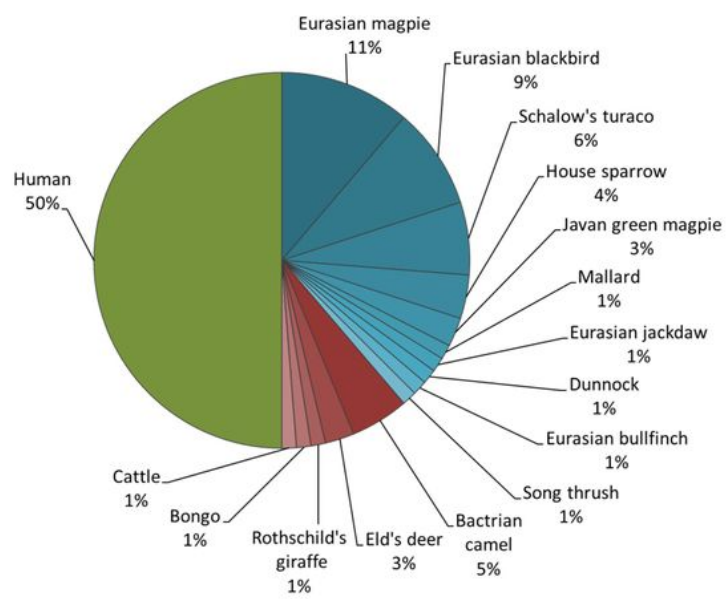

Chester Zoo, $2019(n=20)$

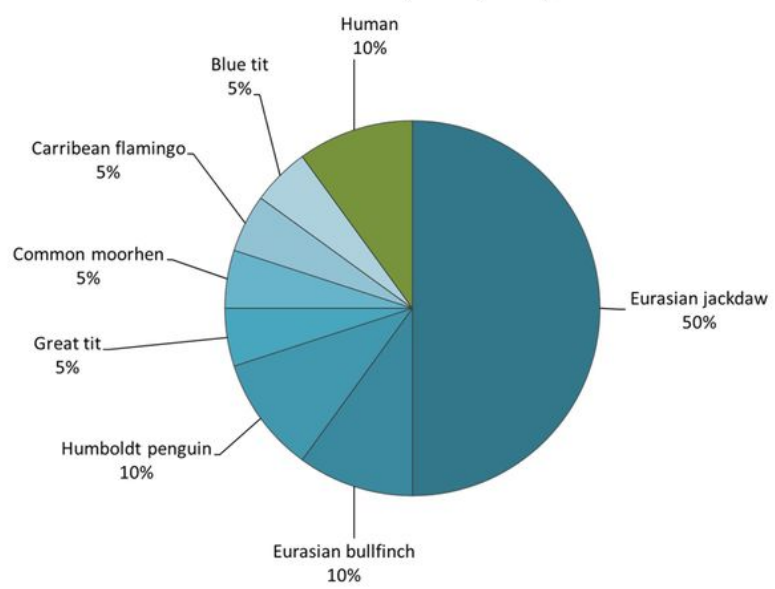

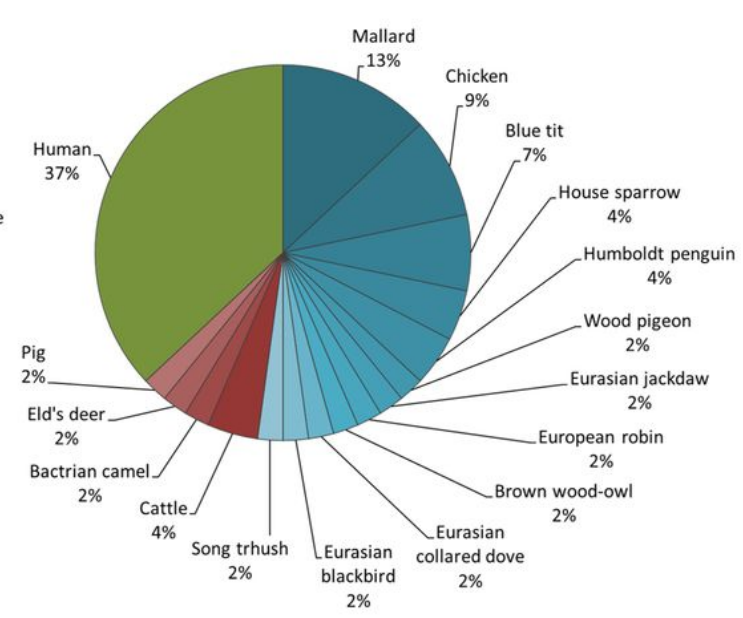

Flamingo Land, $2017(n=19)$

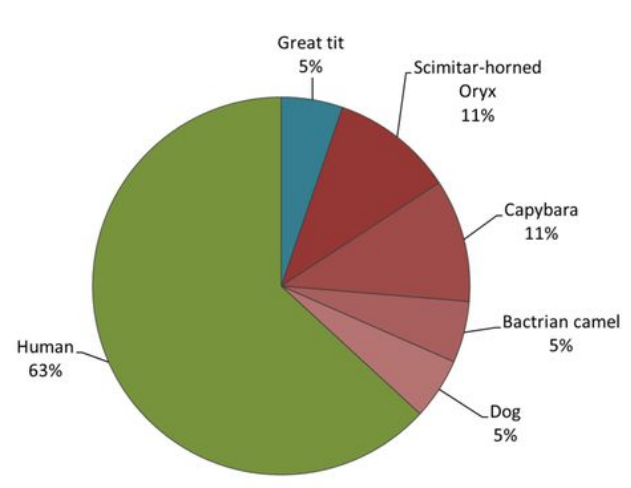

\section{Figure 4}

Host preferences of blood-fed mosquitoes. Birds in shades of blue, non-human mammals in shades of red. 


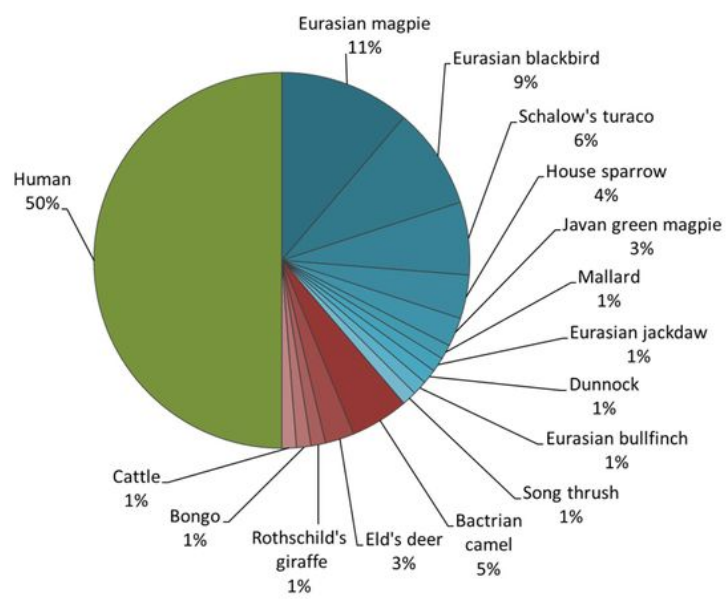

Chester Zoo, $2019(n=20)$

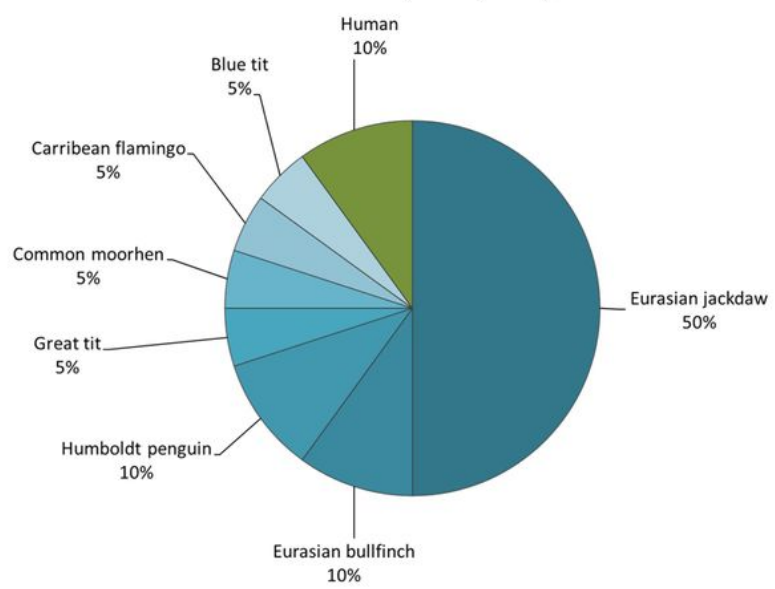

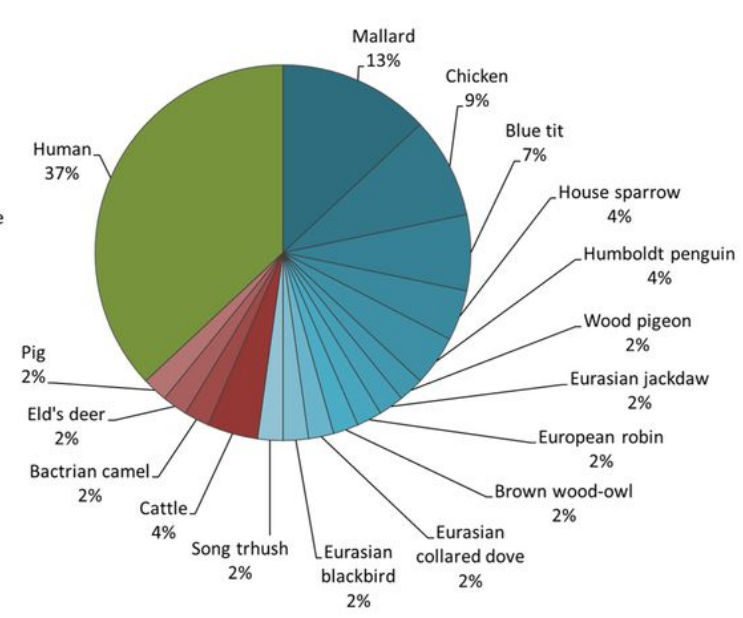

Flamingo Land, $2017(n=19)$

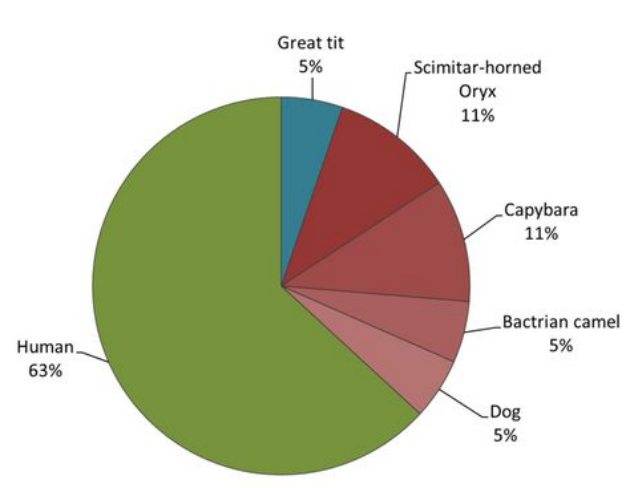

\section{Figure 4}

Host preferences of blood-fed mosquitoes. Birds in shades of blue, non-human mammals in shades of red. 


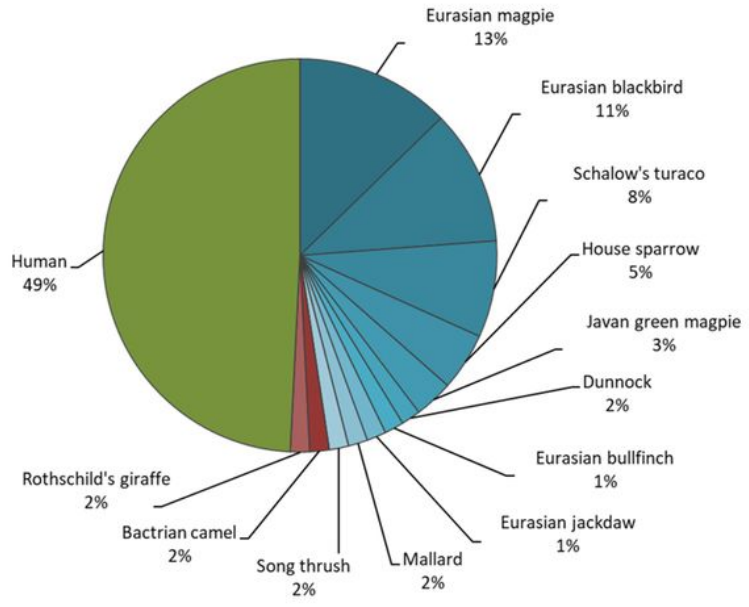

Chester Zoo, 2019 ( $n=18)$

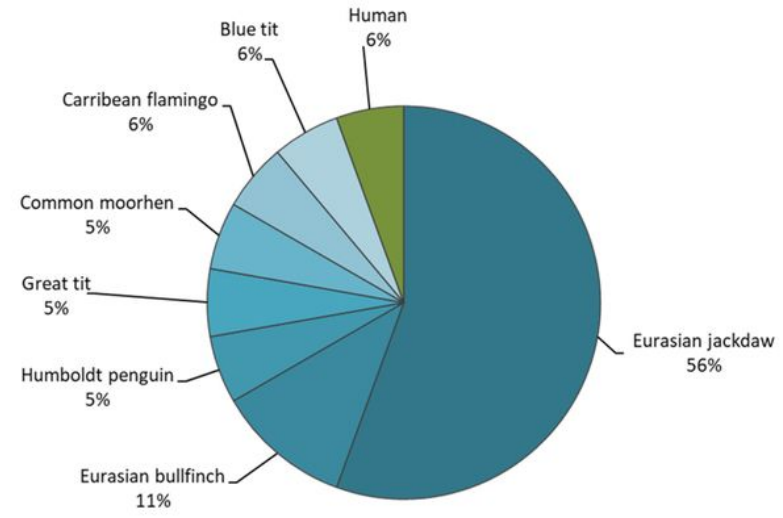

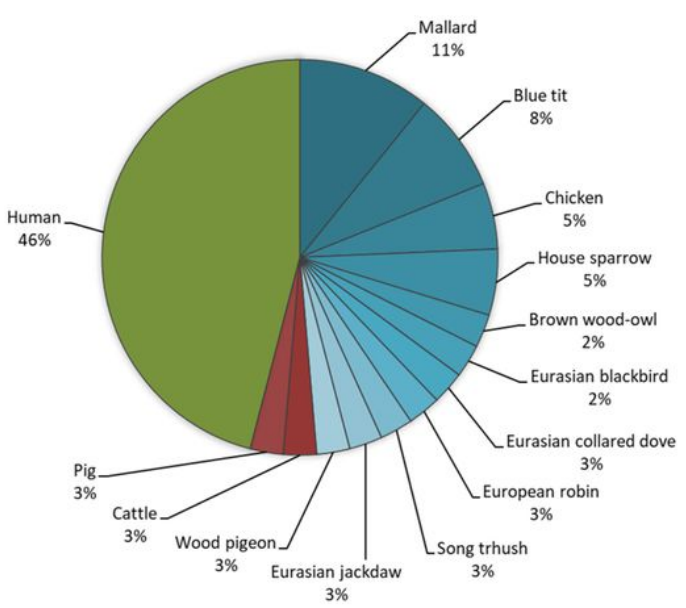

Flamingo Land, $2017(n=12)$

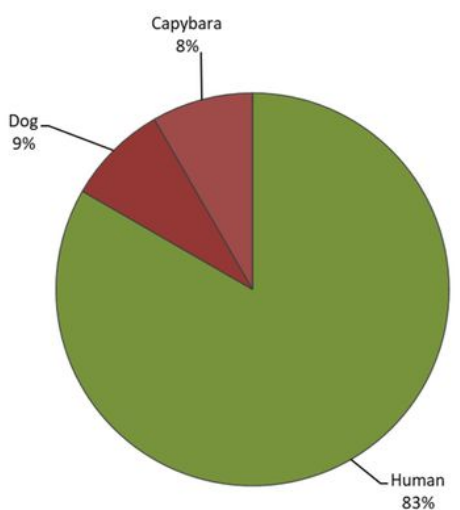

\section{Figure 5}

Host preferences of Culex pipiens. Birds in shades of blue, non-human mammals in shades of red. 


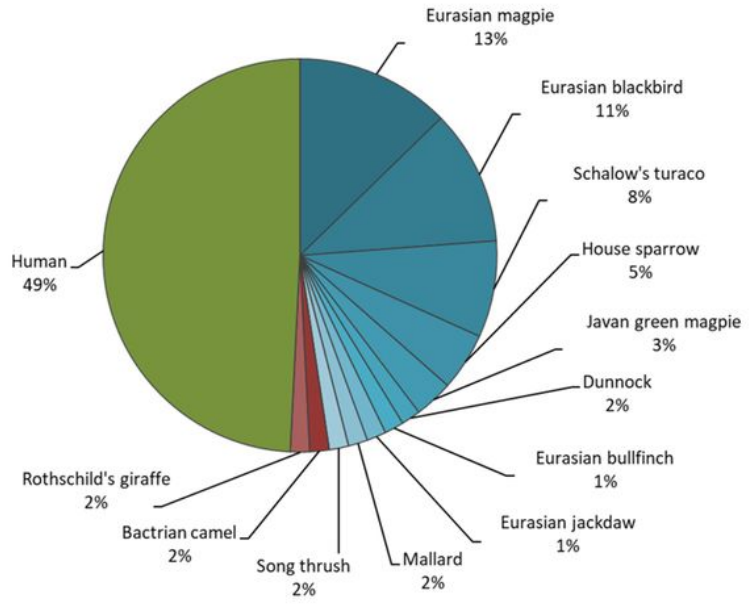

Chester Zoo, 2019 ( $n=18)$

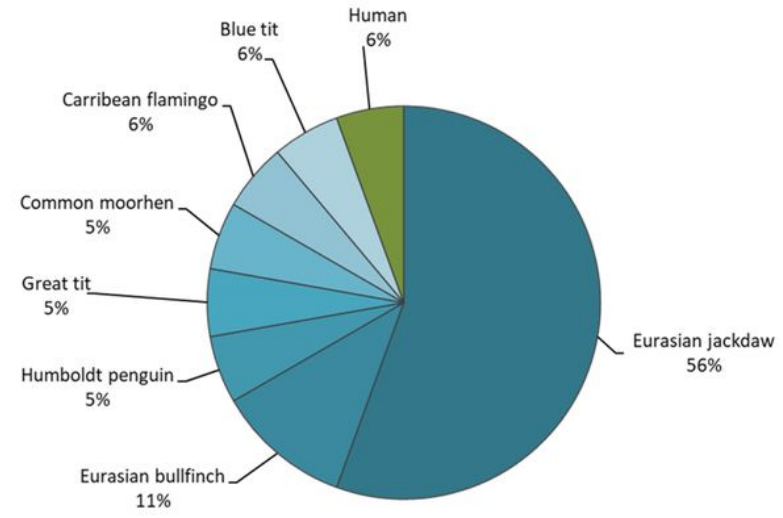

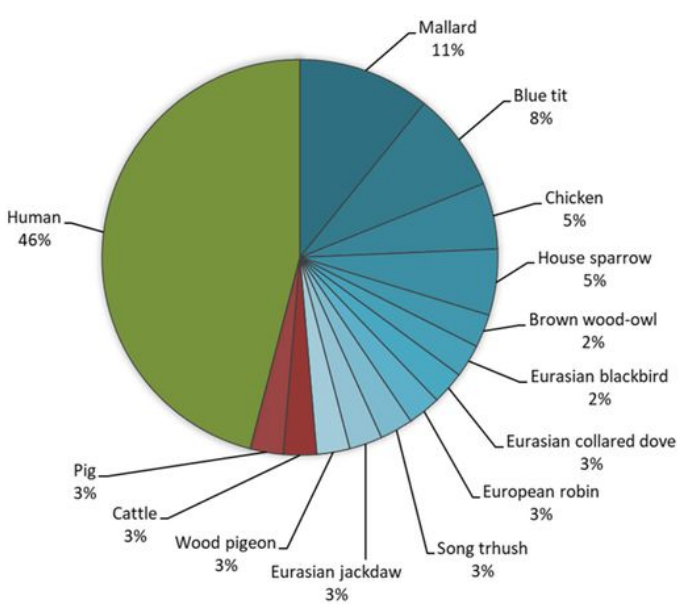

Flamingo Land, $2017(n=12)$

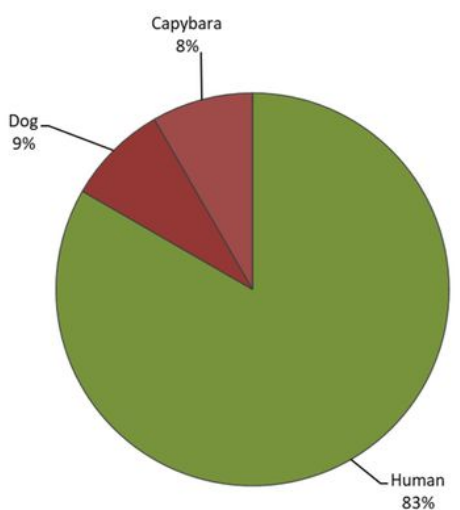

\section{Figure 5}

Host preferences of Culex pipiens. Birds in shades of blue, non-human mammals in shades of red. 


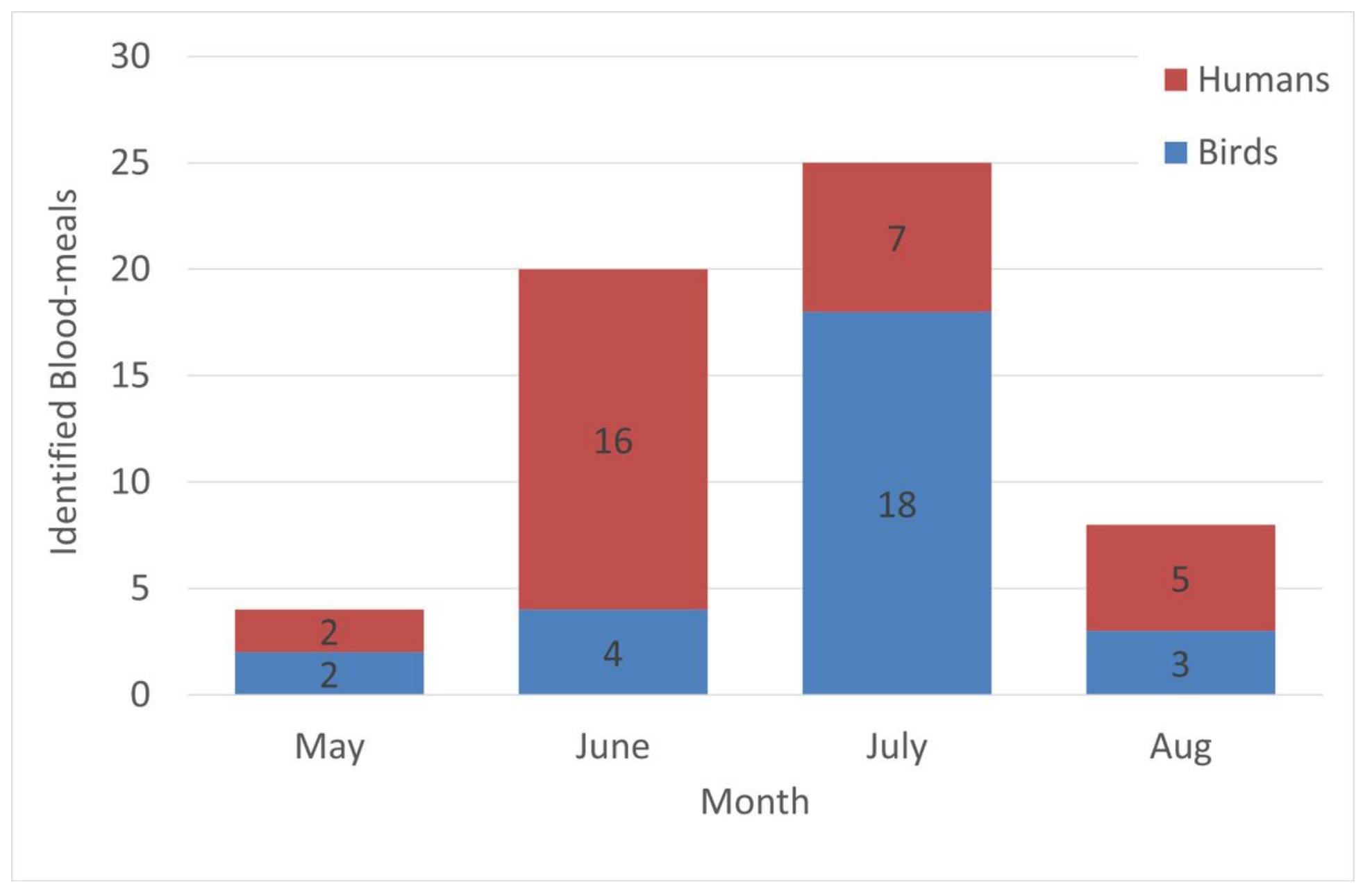

Figure 6

Host preferences of Culex pipiens during 2017 in Chester Zoo. 


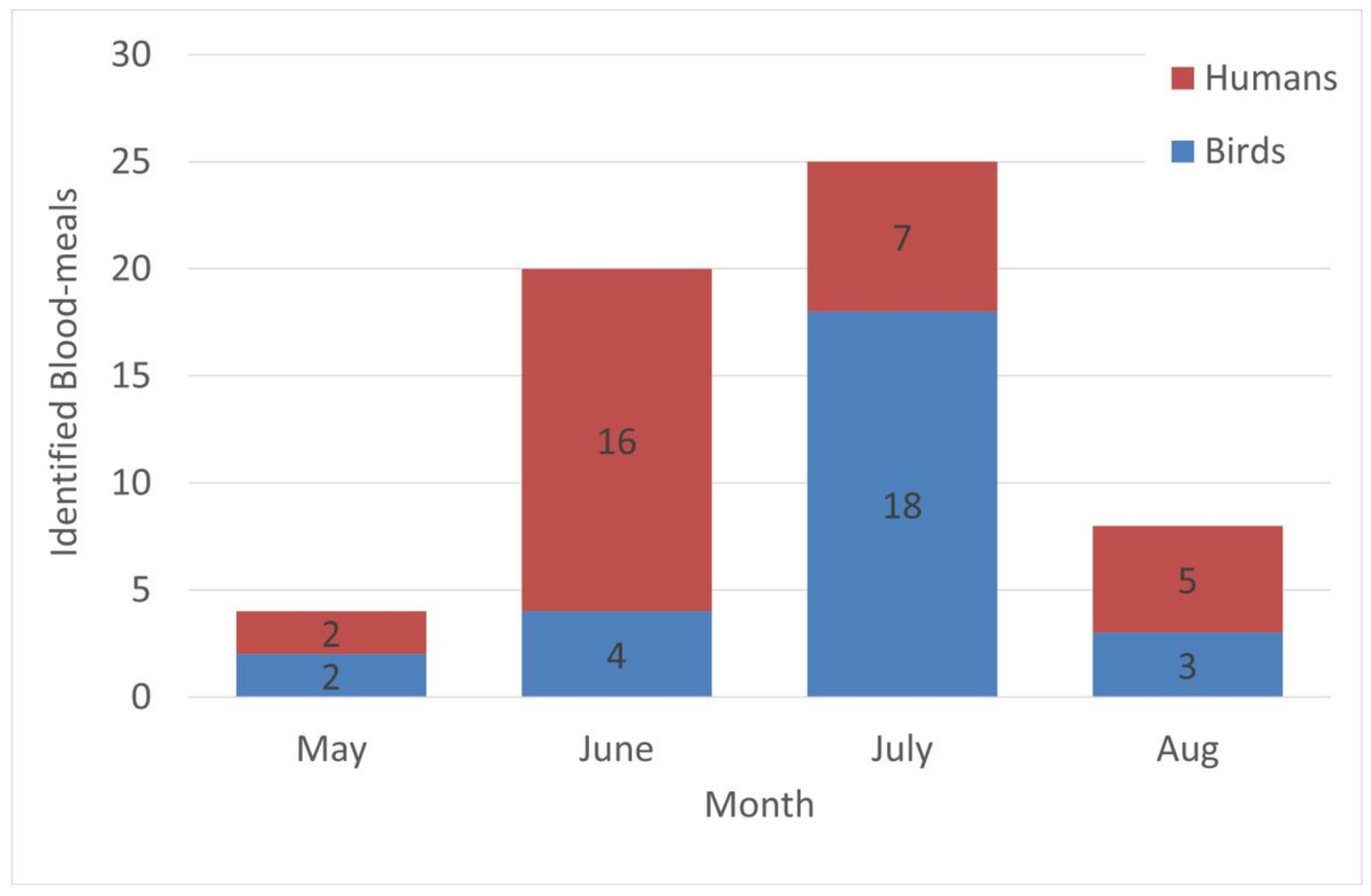

Figure 6

Host preferences of Culex pipiens during 2017 in Chester Zoo. 

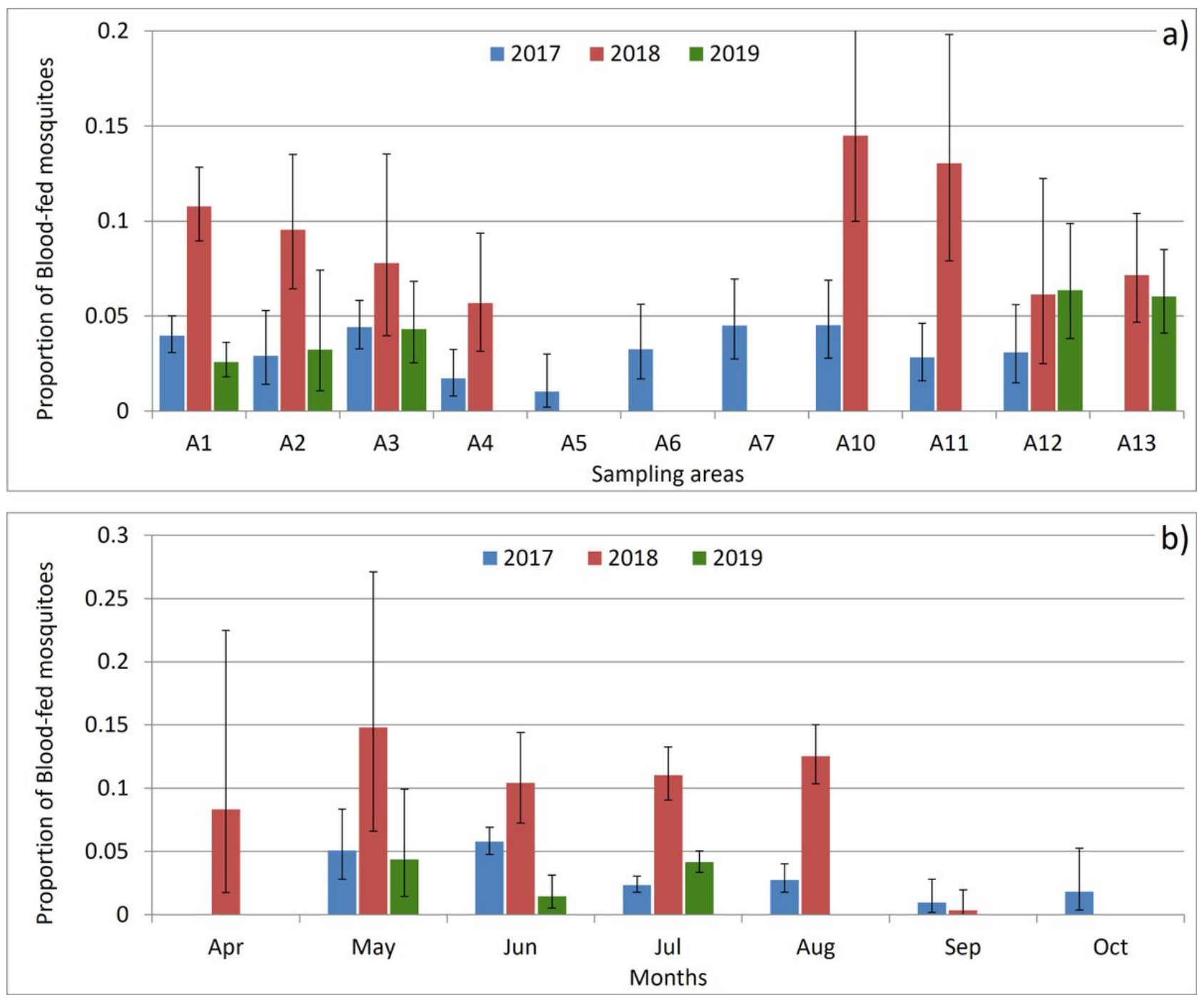

Figure 7

Proportion of blood-fed mosquitoes in Chester Zoo. a) Sampling areas; b) Months. Error bars: 95\% confidence intervals. Sampling areas and months without bars were not sampled in that year. Sampling areas can be consulted in Fig. 2. 

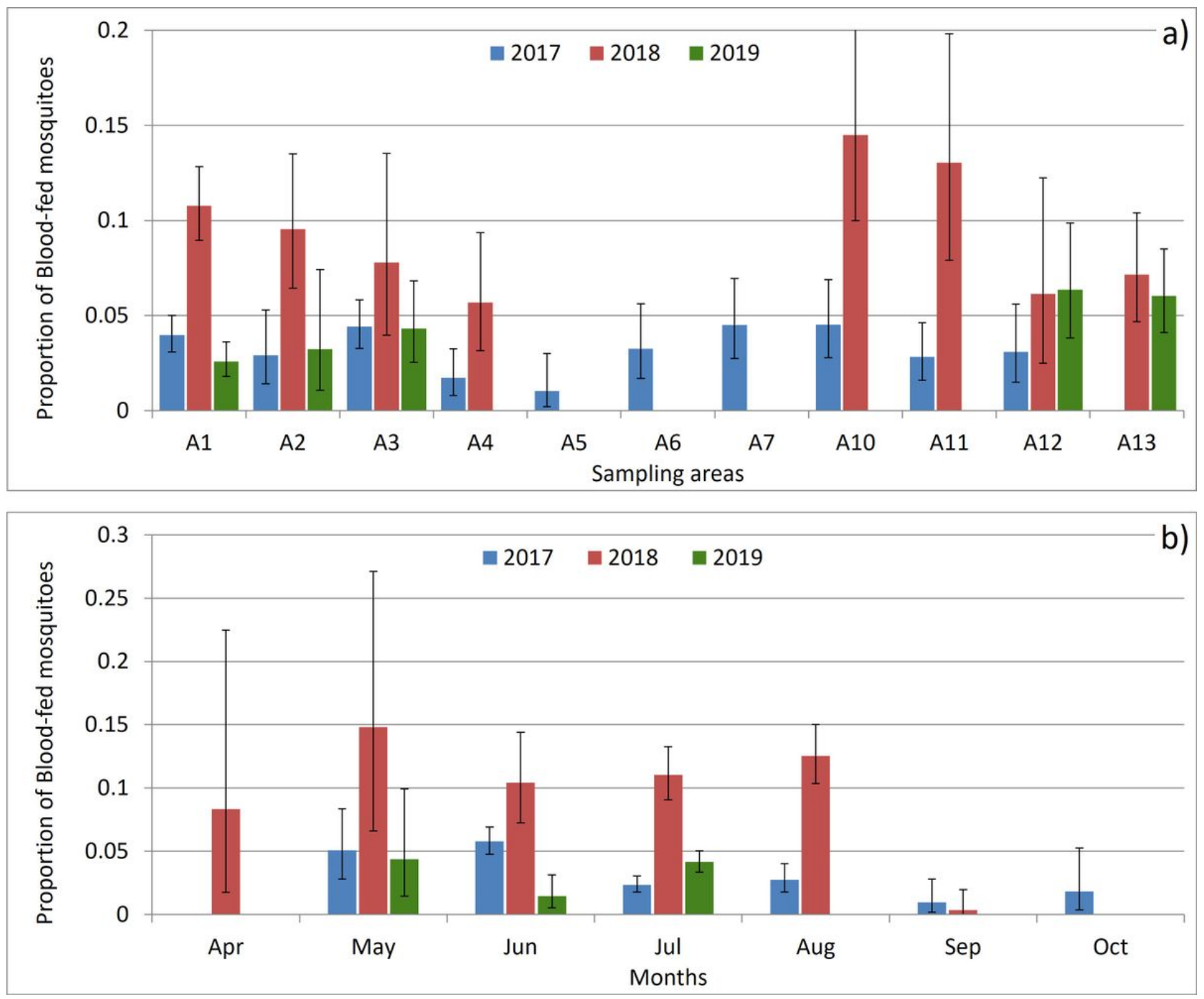

Figure 7

Proportion of blood-fed mosquitoes in Chester Zoo. a) Sampling areas; b) Months. Error bars: 95\% confidence intervals. Sampling areas and months without bars were not sampled in that year. Sampling areas can be consulted in Fig. 2.
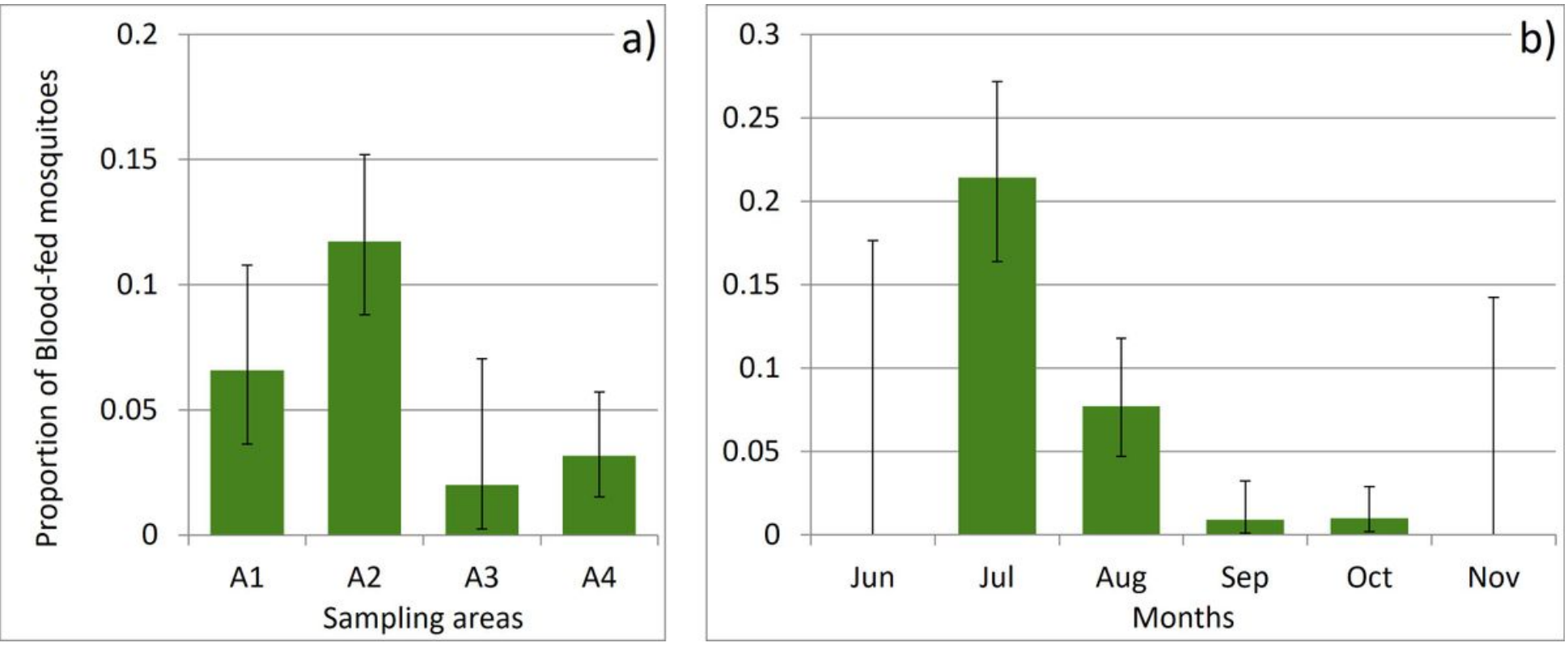

Figure 8 
Proportion of blood-fed mosquitoes in Flamingo Land, 2017. a) Sampling areas; b) Months. Error bars: $95 \%$ confidence intervals. Sampling areas are presented in Fig. 3.
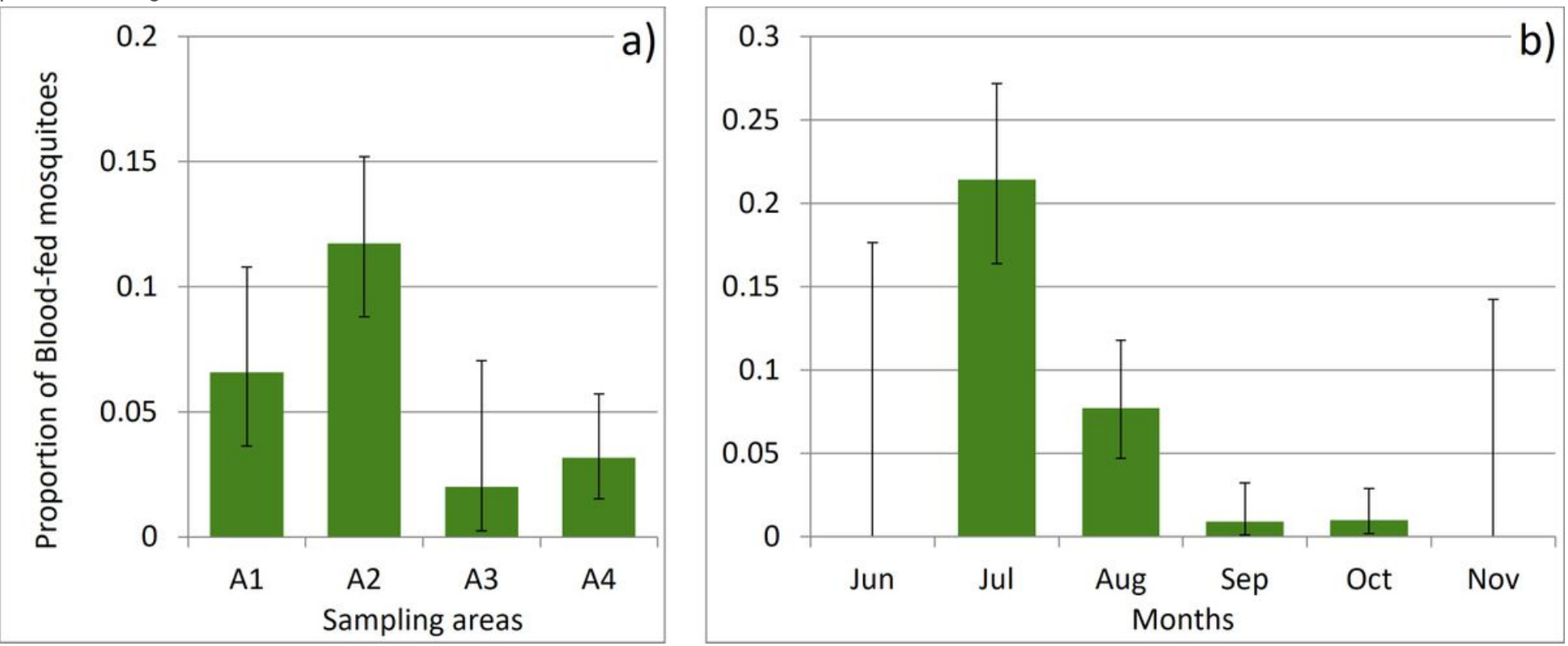

\section{Figure 8}

Proportion of blood-fed mosquitoes in Flamingo Land, 2017. a) Sampling areas; b) Months. Error bars: $95 \%$ confidence intervals. Sampling areas are presented in Fig. 3.

\section{Supplementary Files}

This is a list of supplementary files associated with this preprint. Click to download.

- Additionalfile1.docx

- Additionalfile1.docx

- FigureS1Additionalproportions.jpg

- FigureS1Additionalproportions.jpg

- GraphicAbstract.jpg

- GraphicAbstract.jpg 